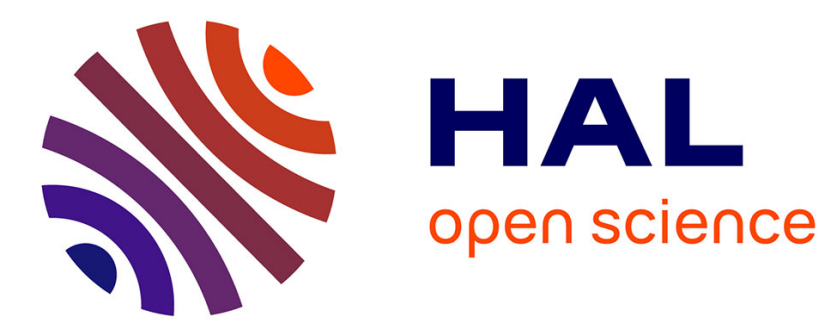

\title{
A Well-Balanced Finite Volume Scheme for a Mixed Hyperbolic/Parabolic System to Model Chemotaxis
}

Christophe Berthon, Anaïs Crestetto, Françoise Foucher

\section{To cite this version:}

Christophe Berthon, Anaïs Crestetto, Françoise Foucher. A Well-Balanced Finite Volume Scheme for a Mixed Hyperbolic/Parabolic System to Model Chemotaxis. Journal of Scientific Computing, 2015, 67, pp.618-643. hal-01620749

\section{HAL Id: hal-01620749 \\ https://hal.science/hal-01620749}

Submitted on 20 Oct 2017

HAL is a multi-disciplinary open access archive for the deposit and dissemination of scientific research documents, whether they are published or not. The documents may come from teaching and research institutions in France or abroad, or from public or private research centers.
L'archive ouverte pluridisciplinaire HAL, est destinée au dépôt et à la diffusion de documents scientifiques de niveau recherche, publiés ou non, émanant des établissements d'enseignement et de recherche français ou étrangers, des laboratoires publics ou privés. 


\title{
A WELL-BALANCED FINITE VOLUME SCHEME FOR A MIXED HYPERBOLIC/PARABOLIC SYSTEM TO MODEL CHEMOTAXIS
}

\author{
CHRISTOPHE BERTHON*, ANAÏS CRESTETTO*, AND FRANÇOISE FOUCHER*†
}

AMS subject classifications. 65M08,65M12, 35L60

Key words. Mixed hyperbolic/parabolic PDE, finite volume method of Godunov type, wellbalanced schemes, asymptotic preserving schemes, chemotaxis model.

\begin{abstract}
This work is concerned by the numerical approximation of the weak solutions of a system of partial differential equations arising when modeling the movements of cells according to a chemoattractant concentration. The adopted PDE system turns out to couple a hyperbolic system with a diffusive equation. The solutions of such a model satisfy several properties to be preserved at the numerical level. Indeed, the solutions may contain vacuum, satisfy steady regimes and asymptotic regimes. By deriving a judicious approximate Riemann solver, a finite volume method is designed in order to exactly preserve the steady regimes of particular physical interest. Moreover, the scheme is able to deal with vacuum regions and it preserves the asymptotic regimes. Numerous numerical experiments illustrate the relevance of the proposed numerical procedure.
\end{abstract}

1. Introduction. The present work concerns the derivation of a finite volume scheme to approximate the weak solutions of the following system of partial differential equations:

$$
\left\{\begin{array}{l}
\partial_{t} \rho+\partial_{x}(\rho u)=0 \\
\partial_{t}(\rho u)+\partial_{x}\left(\rho u^{2}+p(\rho)\right)=\chi \rho \partial_{x} \phi-\alpha \rho u \\
\partial_{t} \phi-D \partial_{x x} \phi=a \rho-b \phi
\end{array}\right.
$$

where the unknown vector $w={ }^{t}(\rho, \rho u, \phi)$ belongs to the following convex set of admissible states:

$$
\Omega=\left\{w={ }^{t}(\rho, \rho u, \phi) \in \mathbb{R}^{3} ; \rho \geq 0, u \in \mathbb{R}, \phi \geq 0\right\} .
$$

This model is clearly in a close relationship with the so-called shallow-water model, or the isentropic Euler equations with friction [17, 23], or autogravitational flow models $[14,15,7,15]$. Here, the system (1.1) enters the modelling of the chemotaxis $[40,51,31]$ where $\rho(x, t)$ denotes the density of endothelial cells, $u(x, t)$ the velocity of the cells and $\phi(x, t)$ the concentration of chemoattractant. Formally, it models the shifting of the cells according to the gradient of the chemical attractor. Simultaneously, the displacement of the cells is decelerated by friction forces with the substratum. The movement due to the chemoattractant is weighted by the non negative constant $\chi$ while $\alpha \geq 0$ represents the strength of the friction forces.

Concerning the evolution of the chemoattractant concentration $\phi$, the positive constants $a$ and $b$ are respectively the production and the consumption rate. Here, since the chemoattractant is produced by the cells, the production term is proportional to the density. The positive constant $D$ stands for the chemoattractant diffusion coefficient.

${ }^{*}$ Laboratoire de Mathématiques Jean Leray, CNRS UMR 6629, Université de Nantes, 2 rue de la Houssinière, BP 92208, 44322 Nantes, France.

†École Centrale de Nantes, 1 rue de la Noë, BP 92101, 44321 Nantes cedex 3, France. 
The model is now closed by selecting a pressure law. In order to avoid overcrowding of the cells, an isentropic gas pressure law [51] is here adopted:

$$
p(\rho)=\delta \rho^{\gamma},
$$

where $\delta>0$ and $\gamma>1$ are given parameters.

The main objective of the present work is to design a finite volume scheme to approximate the weak solutions of (1.1)-(1.3) with a particular attention to the steady states. Non trivial steady states are of prime importance in the numerical simulations. Indeed, most of the numerical experiments of real interest are expected to converge for large time enough to steady states. As a consequence, the derived scheme must accurately approximate these particular solutions.

After the work by Greenberg and LeRoux [39] (see also [13, 33, 43]), the derivation of well-balanced schemes turns out to be a relevant technique to avoid some failures in the correct capture of the steady regimes. During the two last decades, numerous papers were devoted to well-balance strategies with a particular attention to the shallow-water model (see [39, 1, 10, 21, 25, 53, 57, 16] and references therein for a non exhaustive bibliography). The well-balance property is now well-understood as long as the non trivial steady states do not involve strongly nonlinear formulation. For instance, the well-known hydrostatic reconstruction by Audusse et al. [1] is perfectly adapted to exactly capture the celebrate lake at rest for the Saint-Venant system (see $[2,44,10]$ for related works).

Now, the steady states issuing from (1.1) are very severe and usual well-balance strategies fail. Indeed, the steady states associated to (1.1), exhibited in [51] by Natalini et al., are governed by the following PDE system:

$$
\left\{\begin{array}{l}
\partial_{x}(\rho u)=0 \\
\partial_{x}\left(\rho u^{2}+p(\rho)\right)=\chi \rho \partial_{x} \phi-\alpha \rho u \\
-D \partial_{x x} \phi=a \rho-b \phi
\end{array}\right.
$$

Because of some chemical arguments, we are concerning with steady states at rest, i.e. $u=0$. As a consequence, steady states of interest are now solutions of

$$
\left\{\begin{array}{l}
u=0 \\
\partial_{x} p(\rho)=\chi \rho \partial_{x} \phi \\
-D \partial_{x x} \phi=a \rho-b \phi .
\end{array}\right.
$$

Since the pressure is given by (1.3), we obtain the following definition of the steady states:

$$
\begin{aligned}
& u=0 \quad \text { and } \quad e(\rho)-\chi \phi=\mathrm{cst}, \\
& -D \partial_{x x} \phi=a \rho-b \phi
\end{aligned}
$$

where we have set

$$
e(\rho)=\frac{\gamma}{\gamma-1} \delta \rho^{\gamma-1}
$$

Before we detail the stationary behavior of $\phi$, let us comment the relation (1.4a). Indeed, by enforcing $\gamma=2$, we get a steady state defined by $u=0$ and $2 \delta \rho-\chi \phi=$ cst, which coincides with the famous lake at rest. As a consequence, as soon as $\gamma=2$, 
usual well-balanced procedures $[1,13,33]$ can be considered in order to correctly preserve solution satisfying (1.4a). However, the situation turns out to be drastically distinct when considering $\gamma \neq 2$. In the particular case $\gamma=1$, Natalini and Ribot [50] have introduced numerical techniques to increase the order of accuracy for large time simulations and thus to accurately approximate non obvious steady states given by (1.4a) (see also the work by Gosse [34, 35, 37] or by Filbet and Shu [28]). More recently, in [51], Natalini and coauthors have suggested a suitable extension of the hydrostatic reconstruction [1] to deal with nonlinear steady states given by (1.4a) independently of the choice of $\gamma>1$.

Moreover, the numerical scheme introduced in [51] is relevant to approximate vacuum regions. Indeed and similarly to the shallow-water model, vacuum is a natural solution of (1.1)-(1.3). With a positive initial density, vacuum may appear in a finite time. From a numerical point of view, vacuum stays a very delicate problem, which must be carefully considered.

The challenging objective of this paper is to derive a numerical scheme relevant for vacuum but also well-balanced for $C^{1}(\mathbb{R})$ steady states given by both relation (1.4) and not only (1.4a). Now, to consider (1.4), we have to solve (1.4b) with

$$
\rho=e^{-1}(\chi \phi+K), \quad K \text { a given constant. }
$$

Because of the nonlinear form of $e^{-1}$, the equation (1.4b) can be explicitly solved only with $\gamma=2$. The reader is referred to [51] where the system (1.4) is studied. In particular, with $\gamma=2$, Natalini et al. [51] proposed a solution of (1.4b) over a bounded domain. For the sake of simplicity in the forthcoming developments, we give the solution of $(1.4 \mathrm{~b})$ over $\mathbb{R}$. After an easy derivation, the solution of (1.4b) reads, over the domain of non negative chemoattractant concentration $\phi$, as follows:

$$
\left\{\begin{array}{l}
\rho(x)=0 \\
\phi(x)=A \cosh \left(x \sqrt{\frac{b}{D}}\right)+B \sinh \left(x \sqrt{\frac{b}{D}}\right),
\end{array}\right.
$$

or, within the domain of positive density $\rho$ and non negative $\phi$ :

$$
\left\{\begin{array}{l}
\rho(x)=\frac{1}{2 \delta}(\chi \phi(x)+K), \\
\phi(x)=A \cos (x \sqrt{|C|})+B \sin (x \sqrt{|C|})-\frac{K a}{2 \delta b-a \chi} \quad \text { with } C<0,
\end{array}\right.
$$

or

$$
\left\{\begin{array}{l}
\rho(x)=\frac{1}{2 \delta}(\chi \phi(x)+K), \\
\phi(x)=A \cosh (x \sqrt{C})+B \sinh (x \sqrt{C})-\frac{K a}{2 \delta b-a \chi} \quad \text { with } C>0
\end{array}\right.
$$

where $A, B$ and $K$ denote some given constants and

$$
C=\frac{1}{D}\left(b-\frac{a \chi}{2 \delta}\right) .
$$

Equipped with these steady states, the aim of the paper is to derive a wellbalanced scheme able to exactly preserve the relation (1.4a) independently of $\gamma>1$. 
Moreover, as soon as $\gamma=2$, the scheme must exactly capture the full definition of the steady states, namely (1.6) or (1.7) or (1.8). To address such an issue, we suggest to consider Godunov-type methods $[41,32,54]$. In the next section, we derive an approximate Riemann solver to correctly mimic the Riemann solutions associated to (1.1). At this level, two main difficulties must be considered. The first one comes from the parabolic equation. We adopt a reformulation of the chemoattractant evolution equation by introducing a suitable discretization of the space variation of $\phi$. The second one comes from the source terms. Indeed, we propose to enter the source terms within the definition of the approximate Riemann solver. As a consequence, the well-known Harten, Lax and van Leer Theorem [41] cannot be directly applied (see [39, 46, 30, 29]). However, adopting suitable approximations, we exhibit an approximate Riemann solver able to exactly capture the steady states given by (1.4a) for all $\gamma>1$. Moreover, introducing a judicious limitation technique, the developed approximate Riemann solver turns out to be relevant for vacuum solutions. Arguing the obtained approximate Riemann solver, in Section 3 we derive the Godunov-type scheme and we prove its main properties. We establish the robustness and the exact preservation of states according to (1.4a). In addition, we introduce a judicious formula to approximate the independent space variation of $\phi$ in order to also enforce the exact capture of the steady states (1.6), (1.7) and (1.8) as soon as $\gamma=2$. Let us emphasize that the steady state solutions are not fully characterized when $\gamma$ is not equal to 2 because of equation (1.4b). As a consequence, enforcing $\gamma \neq 2$, it is not possible to propose an exact capture of the steady states but just a (1.4a)-capturing scheme. Then, the designed scheme will not be fully well-balanced with $\gamma \neq 2$ but it will give a very good approximation of steady states since it exactly preserves (1.4a). Moreover, the derived scheme satisfies an additional property detailed in Section 4. Indeed, after $[11,36,52]$ (see also $[17,8,12,50,38]$ ), as long as the friction phenomenon is dominant, and for a long time, the system (1.1) admits a particular asymptotic behavior. At least formally, one can show that the solutions of the initial PDE problem (1.1) asymptotically coincide with the solutions of a parabolic diffusive equation. We prove that the here proposed scheme admits a similar asymptotic behavior. More precisely, we establish that, in the limit of an asymptotic parameter to govern the friction coefficient and the long time, the asymptotic behavior of the scheme corresponds to a discretization of the asymptotic parabolic diffusive equation. Section 5 is devoted to illustrate the developed numerical approach and several numerical experiments are performed. A brief conclusion achieves the paper.

2. An approximate Riemann solver. To derive the required numerical scheme, we first introduce a suitable approximate Riemann solver according to the definition proposed by Harten, Lax and van Leer [41]. Let us recall that an approximate Riemann solver, denoted $\tilde{w}\left(x / t ; w_{L}, w_{R}\right)$, is a self-similar function to mimic the exact solution $w_{\mathcal{R}}\left(x, t ; w_{L}, w_{R}\right)$ of the Riemann problem associated to (1.1), i.e. with an initial datum given by

$$
w(x, t=0)= \begin{cases}w_{L} & \text { if } x<0 \\ w_{R} & \text { if } x>0\end{cases}
$$

where $w_{L} \in \Omega$ and $w_{R} \in \Omega$ are two given constant states.

We adopt an approximate Riemann solver made of four constant states separated by three discontinuities, respectively propagating with velocities $\lambda_{L}<0,0$ and $\lambda_{R}>$ 
0. This approximate Riemann solver thus reads as follows:

$$
\tilde{w}\left(x / t ; w_{L}, w_{R}\right)= \begin{cases}w_{L} & \text { if } x<\lambda_{L} t, \\ w_{L}^{\star} & \text { if } \lambda_{L} t<x<0, \\ w_{R}^{\star} & \text { if } 0<x<\lambda_{R} t \\ w_{R} & \text { if } x>\lambda_{R} t .\end{cases}
$$

From now on, the velocities $\lambda_{L}$ and $\lambda_{R}$ must be chosen large enough in order to enforce some robustness properties (see [41]).

In order to evaluate relevant intermediate states, we propose to adopt the integral consistency condition introduced by Harten, Lax and van Leer [41] (for instance, see also $[13,29,48,55])$. As a consequence, we would like $\tilde{w}$ to satisfy

$$
\frac{1}{\Delta x} \int_{-\Delta x / 2}^{\Delta x / 2} \tilde{w}\left(\frac{x}{\Delta t}, w_{L}, w_{R}\right) \mathrm{d} x=\frac{1}{\Delta x} \int_{-\Delta x / 2}^{\Delta x / 2} w_{\mathcal{R}}\left(x, \Delta t ; w_{L}, w_{R}\right) \mathrm{d} x .
$$

From the definition of $\tilde{w}$, given by (2.1), we immediately deduce

$\frac{1}{\Delta x} \int_{-\Delta x / 2}^{\Delta x / 2} \tilde{w}\left(\frac{x}{\Delta t}, w_{L}, w_{R}\right) \mathrm{d} x=\frac{1}{2}\left(w_{L}+w_{R}\right)+\frac{\Delta t}{\Delta x}\left(\lambda_{L}\left(w_{L}-w_{L}^{\star}\right)+\lambda_{R}\left(w_{R}^{\star}-w_{R}\right)\right)$.

The challenging objective is now to correctly evaluate the average of the exact Riemann solution. From now on, let us underline that the exact evaluation of the average of $w_{\mathcal{R}}$ will be unreachable because of the source term. Therefore, in (2.2), the exact Riemann solution $w_{\mathcal{R}}$ will be substituted by a relevant approximation.

For the sake of simplicity in the notations, let us introduce

$$
f(w)=\left(\begin{array}{c}
\rho u \\
\rho u^{2}+p(\rho) \\
-D \partial_{x} \phi
\end{array}\right) \quad \text { and } \quad S(w)=\left(\begin{array}{c}
0 \\
\chi \rho \partial_{x} \phi-\alpha \rho u \\
a \rho-b \phi
\end{array}\right),
$$

so that the system (1.1) rewrites as follows:

$$
\partial_{t} w+\partial_{x} f(w)=S(w) .
$$

Let us note that we have left $\chi \rho \partial_{x} \phi$ within the source term and we have decided that this term does not participate to the flux function $f$. In fact, this is natural since $\phi$ is given by a diffusion equation. Moreover, the source term will be taken into account within the approximate Riemann solver derivation. As a consequence, the product $\rho \partial_{x} \phi$ will be relevantly coupled.

Now, to evaluate the right hand side in (2.2), we integrate (2.4) over $(-\Delta x / 2, \Delta x / 2) \times$ $(0, \Delta t)$ to get

$$
\begin{aligned}
\frac{1}{\Delta x} \int_{-\Delta x / 2}^{\Delta x / 2} w_{\mathcal{R}}\left(x, \Delta t ; w_{L}, w_{R}\right) \mathrm{d} x=\frac{1}{2}\left(w_{L}+w_{R}\right) \\
\quad-\frac{1}{\Delta x} \int_{0}^{\Delta t} f\left(w_{\mathcal{R}}\left(\Delta x / 2, t ; w_{L}, w_{R}\right)\right) d t \\
+\frac{1}{\Delta x} \int_{0}^{\Delta t} f\left(w_{\mathcal{R}}\left(-\Delta x / 2, t ; w_{L}, w_{R}\right)\right) d t \\
+\frac{1}{\Delta x} \int_{-\Delta x / 2}^{\Delta x / 2} \int_{0}^{\Delta t} S\left(w_{\mathcal{R}}\left(x, t ; w_{L}, w_{R}\right)\right) d x d t .
\end{aligned}
$$


At the main discrepancy with usual approximate Riemann solver derivation [41, $32,54]$, because of the source term and the coupling with the diffusion equation, we cannot ensure

$$
w_{\mathcal{R}}\left(-\Delta x / 2, t ; w_{L}, w_{R}\right)=w_{L} \quad \text { and } \quad w_{\mathcal{R}}\left(\Delta x / 2, t ; w_{L}, w_{R}\right)=w_{R}
$$

Such a property here turns out to be wrong independently of $\Delta t$ and $\Delta x$ (see $[4,6$, $5,24]$ ). As a consequence, we adopt the following approximations:

$$
\begin{gathered}
\int_{0}^{\Delta t} f\left(w_{\mathcal{R}}\left(-\Delta x / 2, t ; w_{L}, w_{R}\right)\right) d t \simeq \Delta t\left(\begin{array}{c}
\rho_{L} u_{L} \\
\rho_{L} u_{L}^{2}+p\left(\rho_{L}\right) \\
-D\left(\partial_{x} \phi\right)_{L}
\end{array}\right), \\
\int_{0}^{\Delta t} f\left(w_{\mathcal{R}}\left(\Delta x / 2, t ; w_{L}, w_{R}\right)\right) d t \simeq \Delta t\left(\begin{array}{c}
\rho_{R} u_{R} \\
\rho_{R} u_{R}^{2}+p\left(\rho_{R}\right) \\
-D\left(\partial_{x} \phi\right)_{R}
\end{array}\right),
\end{gathered}
$$

where both $\left(\partial_{x} \phi\right)_{L}$ and $\left(\partial_{x} \phi\right)_{R}$ will be defined later on.

Moreover, we suggest to substitute the source term integral associated to $\rho \partial_{x} \phi$ by a consistent approximation as follows:

$$
\frac{1}{\Delta x} \int_{-\Delta x / 2}^{\Delta x / 2}\left(\rho_{\mathcal{R}} \partial_{x} \phi_{\mathcal{R}}\right)\left(x, \Delta t ; w_{L}, w_{R}\right) \mathrm{d} x \simeq\left\{\rho \partial_{x} \phi\right\}\left(\Delta x, \Delta t ; w_{L}, w_{R}\right) .
$$

This approximation will find a precise definition according to the required well-balance property.

Equipped with these approximations, we propose to consider a suitable approximation, denoted $\tilde{w}_{\mathcal{R}}\left(x, t ; w_{L}, w_{R}\right)$ of the exact Riemann solution. This approximation is determined by considering the averaged relations (2.5) where the above integral approximations are adopted $[29,30]$. As a consequence, we get

$$
\begin{aligned}
& \frac{1}{\Delta x} \int_{-\Delta x / 2}^{\Delta x / 2} \tilde{\rho}_{\mathcal{R}}\left(x, \Delta t ; w_{L}, w_{R}\right) \mathrm{d} x=\frac{1}{2}\left(\rho_{L}+\rho_{R}\right)-\frac{\Delta t}{\Delta x}\left(\rho_{R} u_{R}-\rho_{L} u_{L}\right) \\
& \frac{1}{\Delta x} \int_{-\Delta x / 2}^{\Delta x / 2}(\tilde{\rho u})_{\mathcal{R}}\left(x, \Delta t ; w_{L}, w_{R}\right) \mathrm{d} x=\frac{1}{2}\left(\rho_{L} u_{L}+\rho_{R} u_{R}\right) \\
& \quad-\frac{\Delta t}{\Delta x}\left(\rho_{R} u_{R}^{2}+p\left(\rho_{R}\right)-\rho_{L} u_{L}^{2}-p\left(\rho_{L}\right)\right)+\chi \Delta t\left\{\rho \partial_{x} \phi\right\}\left(\Delta x, \Delta t ; w_{L}, w_{R}\right) \\
& \quad-\frac{\alpha}{\Delta x} \int_{0}^{\Delta t} \int_{-\Delta x / 2}^{\Delta x / 2}(\tilde{\rho u})_{\mathcal{R}}\left(x, t ; w_{L}, w_{R}\right) \mathrm{d} x \mathrm{~d} t, \\
& \frac{1}{\Delta x} \int_{-\Delta x / 2}^{\Delta x / 2} \tilde{\phi}_{\mathcal{R}}\left(x, \Delta t ; w_{L}, w_{R}\right) \mathrm{d} x=\frac{1}{2}\left(\phi_{L}+\phi_{R}\right)+\frac{\Delta t}{\Delta x} D\left(\left(\partial_{x} \phi\right)_{R}-\left(\partial_{x} \phi\right)_{L}\right) \\
& \quad+\frac{a}{\Delta x} \int_{0}^{\Delta t} \int_{-\Delta x / 2}^{\Delta x / 2} \tilde{\rho}_{\mathcal{R}}\left(x, t ; w_{L}, w_{R}\right) \mathrm{d} x \mathrm{~d} t-\frac{b}{\Delta x} \int_{0}^{\Delta t} \int_{-\Delta x / 2}^{\Delta x / 2} \tilde{\phi}_{\mathcal{R}}\left(x, t ; w_{L}, w_{R}\right) \mathrm{d} x \mathrm{~d} t
\end{aligned}
$$

In fact, it is possible to exhibit explicit formulae for these integrals of interest.

LEMMA 2.1. Let us assume that the approximation $\left\{\rho \partial_{x} \phi\right\}\left(\Delta x, \Delta t ; w_{L}, w_{R}\right)$ does 
not depend on $\Delta t$. Then we have

$$
\begin{aligned}
& \frac{1}{\Delta x} \int_{-\Delta x / 2}^{\Delta x / 2}(\tilde{\rho u})_{\mathcal{R}}\left(x, \Delta t ; w_{L}, w_{R}\right) \mathrm{d} x=\frac{\mathrm{e}^{-\alpha \Delta t}}{2}\left(\rho_{L} u_{L}+\rho_{R} u_{R}\right) \\
& \quad-\frac{1-\mathrm{e}^{-\alpha \Delta t}}{\alpha \Delta x}\left(\rho_{R} u_{R}^{2}+p\left(\rho_{R}\right)-\rho_{L} u_{L}^{2}-p\left(\rho_{L}\right)\right)+\chi \frac{1-\mathrm{e}^{-\alpha \Delta t}}{\alpha}\left\{\rho \partial_{x} \phi\right\}, \\
& \frac{1}{\Delta x} \int_{-\Delta x / 2}^{\Delta x / 2} \tilde{\phi}_{\mathcal{R}}\left(x, \Delta t ; w_{L}, w_{R}\right) \mathrm{d} x=\frac{\mathrm{e}^{-b \Delta t}}{2}\left(\phi_{L}+\phi_{R}\right)+\frac{a}{2} \frac{1-\mathrm{e}^{-b \Delta t}}{b}\left(\rho_{L}+\rho_{R}\right) \\
& \quad+D \frac{1-\mathrm{e}^{-b \Delta t}}{b \Delta x}\left(\left(\partial_{x} \phi\right)_{R}-\left(\partial_{x} \phi\right)_{L}\right)+\frac{a}{b \Delta x}\left(\frac{1-\mathrm{e}^{-b \Delta t}}{b}-\Delta t\right)\left(\rho_{R} u_{R}-\rho_{L} u_{L}\right) .
\end{aligned}
$$

Proof. To evaluate the first integral, momentarily let us introduce the following smooth function:

$$
\mathcal{F}(t)=\frac{1}{\Delta x} \int_{-\Delta x / 2}^{\Delta x / 2}(\tilde{\rho u})_{\mathcal{R}}\left(x, t ; w_{L}, w_{R}\right) \mathrm{d} x
$$

so that the identity $(2.7)$ rewrites

$$
\begin{aligned}
\mathcal{F}(\Delta t)= & \frac{1}{2}\left(\rho_{L} u_{L}+\rho_{R} u_{R}\right)-\frac{\Delta t}{\Delta x}\left(\rho_{R} u_{R}^{2}+p\left(\rho_{R}\right)-\rho_{L} u_{L}^{2}-p\left(\rho_{L}\right)\right) \\
& +\Delta t \chi\left\{\rho \partial_{x} \phi\right\}-\alpha \int_{0}^{\Delta t} \mathcal{F}(t) \mathrm{d} t .
\end{aligned}
$$

Now, we solve this integral equation. Since $\left\{\rho \partial_{x} \phi\right\}$ does not depend on $\Delta t$, the derivative with respect to $\Delta t$ of the above function gives:

$$
\mathcal{F}^{\prime}(\Delta t)=-\frac{1}{\Delta x}\left(\rho_{R} u_{R}^{2}+p\left(\rho_{R}\right)-\rho_{L} u_{L}^{2}-p\left(\rho_{L}\right)\right)+\chi\left\{\rho \partial_{x} \phi\right\}-\alpha \mathcal{F}(\Delta t) .
$$

We easily get

$$
\mathcal{F}(\Delta t)=\Gamma e^{-\alpha \Delta t}+\frac{1}{\alpha}\left(-\frac{1}{\Delta x}\left(\rho_{R} u_{R}^{2}+p\left(\rho_{R}\right)-\rho_{L} u_{L}^{2}-p\left(\rho_{L}\right)\right)+\chi\left\{\rho \partial_{x} \phi\right\}\right)
$$

where $\Gamma$ is a constant directly determined by plugging (2.12) inside (2.11). The first integral (2.9) is thus obtained.

Concerning the second integral, we adopt the same technique. We set

$$
\mathcal{G}(t)=\frac{1}{\Delta x} \int_{-\Delta x / 2}^{\Delta x / 2} \tilde{\phi}_{\mathcal{R}}\left(x, t ; w_{L}, w_{R}\right) \mathrm{d} x
$$

to rewrite (2.8) as follows:

$$
\begin{aligned}
\mathcal{G}(\Delta t)= & \frac{1}{2}\left(\phi_{L}+\phi_{R}\right)+\frac{\Delta t}{\Delta x} D\left(\left(\partial_{x} \phi\right)_{R}-\left(\partial_{x} \phi\right)_{L}\right) \\
& +\frac{a}{\Delta x} \int_{0}^{\Delta t} \int_{-\Delta x / 2}^{\Delta x / 2} \tilde{\rho}_{\mathcal{R}}\left(x, t ; w_{L}, w_{R}\right) \mathrm{d} x \mathrm{~d} t-b \int_{0}^{\Delta t} \mathcal{G}(t) \mathrm{d} t .
\end{aligned}
$$


But, arguing the identity (2.6), we have

$$
\begin{aligned}
\frac{1}{\Delta x} \int_{0}^{\Delta t} \int_{-\Delta x / 2}^{\Delta x / 2} \tilde{\rho}_{\mathcal{R}}\left(x, t ; w_{L}, w_{R}\right) \mathrm{d} x \mathrm{~d} t & =\int_{0}^{\Delta t}\left(\frac{1}{2}\left(\rho_{L}+\rho_{R}\right)-\frac{t}{\Delta x}\left(\rho_{R} u_{R}-\rho_{L} u_{L}\right)\right) \mathrm{d} t \\
& =\frac{\Delta t}{2}\left(\rho_{L}+\rho_{R}\right)-\frac{\Delta t^{2}}{2 \Delta x}\left(\rho_{R} u_{R}-\rho_{L} u_{L}\right) .
\end{aligned}
$$

As a consequence, $\mathcal{G}$ is solution of the following integral equation:

$$
\begin{aligned}
\mathcal{G}(\Delta t)= & \frac{1}{2}\left(\phi_{L}+\phi_{R}\right)+D \frac{\Delta t}{\Delta x}\left(\left(\partial_{x} \phi\right)_{R}-\left(\partial_{x} \phi\right)_{L}\right) \\
& +a\left(\frac{\Delta t}{2}\left(\rho_{L}+\rho_{R}\right)-\frac{\Delta t^{2}}{2 \Delta x}\left(\rho_{R} u_{R}-\rho_{L} u_{L}\right)\right)-b \int_{0}^{\Delta t} \mathcal{G}(t) \mathrm{d} t
\end{aligned}
$$

which is straightforwardly solved to obtain (2.10). The proof is thus completed.

With these approximations of averages of the exact Riemann solution, we now evaluate the approximate Riemann solver $\tilde{w}$. It is made of six unknowns; namely $w_{L}^{\star}={ }^{t}\left(\rho_{L}^{\star}, \rho_{L}^{\star} u_{L}^{\star}, \phi_{L}^{\star}\right)$ and $w_{R}^{\star}={ }^{t}\left(\rho_{R}^{\star}, \rho_{R}^{\star} u_{R}^{\star}, \phi_{R}^{\star}\right)$. As a consequence, we need six independent relations to solve this problem.

Instead of the exact consistency condition (2.2), we impose the following approximate consistency relation:

$$
\frac{1}{\Delta x} \int_{-\Delta x / 2}^{\Delta x / 2} \tilde{w}\left(\frac{x}{\Delta t} ; w_{L}, w_{R}\right) \mathrm{d} x=\frac{1}{\Delta x} \int_{-\Delta x / 2}^{\Delta x / 2} \tilde{w}_{\mathcal{R}}\left(x, \Delta t ; w_{L}, w_{R}\right) \mathrm{d} x
$$

to obtain the following system made of three equations:

$$
\begin{aligned}
& \lambda_{R}\left(\rho_{R}^{\star}-\rho_{R}\right)-\lambda_{L}\left(\rho_{L}^{\star}-\rho_{L}\right)=\rho_{L} u_{L}-\rho_{R} u_{R} \\
& \lambda_{R}\left(\rho_{R}^{\star} u_{R}^{\star}-\rho_{R} u_{R}\right)-\lambda_{L}\left(\rho_{L}^{\star} u_{L}^{\star}-\rho_{L} u_{L}\right)= \\
& \quad \frac{e^{-\alpha \Delta t}-1}{\alpha \Delta t}\left(\left(\rho_{R} u_{R}^{2}+p\left(\rho_{R}\right)-\rho_{L} u_{L}^{2}-p\left(\rho_{L}\right)\right)-\chi \Delta x\left\{\rho \partial_{x} \phi\right\}+\frac{\alpha \Delta x}{2}\left(\rho_{R} u_{R}+\rho_{L} u_{L}\right)\right) \\
& \lambda_{R}\left(\phi_{R}^{\star}-\phi_{R}\right)-\lambda_{L}\left(\phi_{L}^{\star}-\phi_{L}\right)=\mathcal{E}_{0}
\end{aligned}
$$

where, to simplify the notations, we have set

$$
\begin{aligned}
\mathcal{E}_{0}= & \left(\frac{1-\mathrm{e}^{-b \Delta t}}{b \Delta t}\right)\left(D\left(\left(\partial_{x} \phi\right)_{R}-\left(\partial_{x} \phi\right)_{L}\right)-\frac{b \Delta x}{2}\left(\phi_{L}+\phi_{R}\right)+\frac{a \Delta x}{2}\left(\rho_{L}+\rho_{R}\right)\right) \\
& +\frac{a}{b}\left(\frac{1-\mathrm{e}^{-b \Delta t}}{b \Delta t}-1\right)\left(\rho_{R} u_{R}-\rho_{L} u_{L}\right) .
\end{aligned}
$$

At this level, we notice that three equations are missing. Moreover, the definition of $\left\{\rho \partial_{x} \phi\right\}$ must be specified. Actually, we select additional relations in order to enforce the well-balance property.

Concerning the definition of $\left\{\rho \partial_{x} \phi\right\}\left(\Delta x, \Delta t ; w_{L}, w_{R}\right)$, we have the following result:

LEMma 2.2. Assume $w_{L}$ and $w_{R}$ such that the steady state definition (1.4a) holds true:

$$
u_{L}=u_{R}=0 \quad \text { and } \quad e\left(\rho_{L}\right)-\chi \phi_{L}=e\left(\rho_{R}\right)-\chi \phi_{R}
$$


where $e(\rho)$ is given by (1.5).

To enforce the approximate Riemann solver to be stationary, i.e. $w_{L}^{\star}=w_{L}$ and $w_{R}^{\star}=w_{R}$, necessarily we have

$$
\left\{\rho \partial_{x} \phi\right\}\left(\Delta x, \Delta t ; w_{L}, w_{R}\right)=\frac{1}{\chi \Delta x}\left(p\left(\rho_{R}\right)-p\left(\rho_{L}\right)\right)
$$

Proof. Since we enforce the approximate Riemann solver $\tilde{w}$ to be stationary, we have

$$
\rho_{L}^{\star}=\rho_{L}, \quad \rho_{R}^{\star}=\rho_{R}, \quad u_{L}^{\star}=u_{R}^{\star}=u_{L}=u_{R}=0 .
$$

As a consequence, (2.14) now reads

$$
\chi \Delta x\left\{\rho \partial_{x} \phi\right\}\left(\Delta x, \Delta t ; w_{L}, w_{R}\right)-\left(p\left(\rho_{R}\right)-p\left(\rho_{L}\right)\right)=0,
$$

and the proof is achieved.

Arguing the above result, a possible definition of $\left\{\rho \partial_{x} \phi\right\}$ is given as follows:

$$
\left\{\rho \partial_{x} \phi\right\}\left(\Delta x, \Delta t ; w_{L}, w_{R}\right)=\frac{p\left(\rho_{R}\right)-p\left(\rho_{L}\right)}{e\left(\rho_{R}\right)-e\left(\rho_{L}\right)} \frac{\phi_{R}-\phi_{L}}{\Delta x}
$$

This formula is obviously consistent with $\rho \partial_{x} \phi$ since we have

$$
\lim _{\rho_{L, R} \rightarrow \rho} \frac{p\left(\rho_{R}\right)-p\left(\rho_{L}\right)}{e\left(\rho_{R}\right)-e\left(\rho_{L}\right)}=\rho \quad \forall \rho>0
$$

Moreover, according to Lemma 2.1, this discretization of the source term is free from the time step $\Delta t$.

In addition, as soon as $w_{L}$ and $w_{R}$ satisfy (1.4a), we get

$$
\chi\left(\phi_{R}-\phi_{L}\right)=e\left(\rho_{R}\right)-e\left(\rho_{L}\right)
$$

and then the condition (2.17) holds true.

Next, concerning the three missing equations, we first notice that $\rho u$ and $e(\rho)-\chi \phi$ are natural invariant accross the stationary wave (see $[13,39,45]$ ). As a consequence, we suggest to impose the continuity of the momentum across the stationary wave:

$$
\rho_{L}^{\star} u_{L}^{\star}=\rho_{R}^{\star} u_{R}^{\star}
$$

Concerning the second invariant, because of the nonlinear function $e$, we propose to consider the following linearization:

$$
e\left(\rho_{L}\right) \frac{\rho_{L}^{\star}}{\rho_{L}}-\chi \phi_{L}=e\left(\rho_{R}\right) \frac{\rho_{R}^{\star}}{\rho_{R}}-\chi \phi_{R}
$$

According to the diffusion equation, the last missing relation is obtained by preserving the variations of $\phi$ as follows:

$$
\phi_{L}-\phi_{L}^{\star}=\phi_{R}-\phi_{R}^{\star}
$$


By solving the system (2.13)-(2.14)-(2.15)-(2.19)-(2.20)-(2.21), the intermediate states are given by

$$
\begin{aligned}
\rho_{L}^{\star}=\rho_{L} & -\frac{e\left(\rho_{R}\right) / \rho_{R}}{\lambda_{R} e\left(\rho_{L}\right) / \rho_{L}-\lambda_{L} e\left(\rho_{R}\right) / \rho_{R}}\left(\rho_{R} u_{R}-\rho_{L} u_{L}\right) \\
& +\frac{\lambda_{R}}{\lambda_{R} e\left(\rho_{L}\right) / \rho_{L}-\lambda_{L} e\left(\rho_{R}\right) / \rho_{R}}\left(\left(e\left(\rho_{R}\right)-e\left(\rho_{L}\right)\right)-\chi\left(\phi_{R}-\phi_{L}\right)\right), \\
\rho_{R}^{\star}=\rho_{R} & -\frac{e\left(\rho_{L}\right) / \rho_{L}}{\lambda_{R} e\left(\rho_{L}\right) / \rho_{L}-\lambda_{L} e\left(\rho_{R}\right) / \rho_{R}}\left(\rho_{R} u_{R}-\rho_{L} u_{L}\right) \\
& +\frac{\lambda_{L}}{\lambda_{R} e\left(\rho_{L}\right) / \rho_{L}-\lambda_{L} e\left(\rho_{R}\right) / \rho_{R}}\left(\left(e\left(\rho_{R}\right)-e\left(\rho_{L}\right)\right)-\chi\left(\phi_{R}-\phi_{L}\right)\right), \\
q^{\star}=\rho_{L}^{\star} u_{L}^{\star}=\rho_{R}^{\star} u_{R}^{\star}=\frac{\lambda_{R} \rho_{R} u_{R}-\lambda_{L} \rho_{L} u_{L}}{\lambda_{R}-\lambda_{L}} & \left.\quad-\chi \Delta x\left\{\rho \partial_{x} \phi\right\}+\frac{\alpha \Delta x}{2}\left(\rho_{R} u_{R}+\rho_{L} u_{L}\right)\right), \\
& +\frac{e^{-\alpha \Delta t}-1}{\alpha \Delta t\left(\lambda_{R}-\lambda_{L}\right)}\left(\left(\rho_{R} u_{R}^{2}+p\left(\rho_{R}\right)-\rho_{L} u_{L}^{2}-p\left(\rho_{L}\right)\right)\right. \\
\phi_{L}^{\star}=\phi_{L}+ & \frac{\mathcal{E}_{0}}{\lambda_{R}-\lambda_{L}} \quad \text { and } \quad \phi_{R}^{\star}=\phi_{R}+\frac{\mathcal{E}_{0}}{\lambda_{R}-\lambda_{L}},
\end{aligned}
$$

where $\mathcal{E}_{0}$ is defined by $(2.16)$.

In fact, the derived intermediate states are not fully adapted to perform all the simulations of physical interest. Indeed, the vacuum turns out to be a natural solution of (1.1). As a consequence, the proposed approximate Riemann solver must be relevant to deal with vanishing density and/or chemoattractant. To address such an issue, several strategies are based on a suitable choice of $\lambda_{L}$ and $\lambda_{R}$ (for instance, see $[13,1,10,49,18])$. Unfortunately, such an approach is not relevant here. In order to convince the reader, let us consider densities $\rho_{L}$ and $\rho_{R}$ in a neighbourhood of zero. Then, the intermediate densities are governed by $\phi_{R}-\phi_{L}$. As a consequence, the positiveness of the intermediate densities must be imposed in a strong way. We thus adopt a recent consistent cut-off introduced in [3]. First, let us introduce

$$
\rho^{\mathrm{HLL}}=\frac{\lambda_{R} \rho_{R}-\lambda_{L} \rho_{L}}{\lambda_{R}-\lambda_{L}}-\frac{1}{\lambda_{R}-\lambda_{L}}\left(\rho_{R} u_{R}-\rho_{L} u_{L}\right) .
$$

Since $\lambda_{L}<0<\lambda_{R}$, it is always possible to chose $\lambda_{L}$ and $\lambda_{R}$ such that $\rho^{\mathrm{HLL}} \geq 0$. Next, we remark that the equation (2.13) rewrites

$$
\lambda_{R}\left(\rho_{R}^{\star}-\rho^{\mathrm{HLL}}\right)-\lambda_{L}\left(\rho_{L}^{\star}-\rho^{\mathrm{HLL}}\right)=0 .
$$

As soon as both $\rho_{L}^{\star}$ and $\rho_{R}^{\star}$ are non negative, necessarily we have

$$
0 \leq \rho_{L}^{\star} \leq \frac{\lambda_{R}-\lambda_{L}}{-\lambda_{L}} \rho^{\mathrm{HLL}} \quad \text { and } \quad 0 \leq \rho_{R}^{\star} \leq \frac{\lambda_{R}-\lambda_{L}}{\lambda_{R}} \rho^{\mathrm{HLL}} .
$$

Hence, we suggest to modify the intermediate densities (2.22)-(2.23) as follows:

$$
\begin{aligned}
& \rho_{L}^{\star \star}=\min \left(\max \left(0, \rho_{L}^{\star}\right), \frac{\lambda_{R}-\lambda_{L}}{-\lambda_{L}} \rho^{\mathrm{HLL}}\right), \\
& \rho_{R}^{\star \star}=\min \left(\max \left(0, \rho_{R}^{\star}\right), \frac{\lambda_{R}-\lambda_{L}}{\lambda_{R}} \rho^{\mathrm{HLL}}\right),
\end{aligned}
$$


where $\rho^{\mathrm{HLL}} \geq 0$ is defined by $(2.26)$.

From now on, let us emphasize that such a cut-off does not perturb the conservation of the density since (2.13), or equivalently (2.27), holds true.

Concerning the chemoattractant concentration, let us set

$$
\bar{\phi}=\frac{\lambda_{R} \phi_{R}-\lambda_{L} \phi_{L}}{\lambda_{R}-\lambda_{L}}+\frac{1}{\lambda_{R}-\lambda_{L}} \mathcal{E}_{0}
$$

where $\mathcal{E}_{0}$ is given by $(2.16)$. Let us notice that $\bar{\phi}$ stays non negative. Indeed, we have the following statement:

Lemma 2.3. Let $w_{L}$ and $w_{R}$ be two constant states in $\Omega$, defined by (1.2). Assume that the momentum vanishes within the vacuum regions:

$$
\rho_{L} u_{L}=0 \quad \text { if } \rho_{L}=0 \quad \text { and } \quad \rho_{R} u_{R}=0 \quad \text { if } \rho_{R}=0 \text {. }
$$

Moreover, we impose the following convention: if $\phi_{L}=\phi_{R}=0$ then

$$
\left(\partial_{x} \phi\right)_{L} \leq 0 \quad \text { and } \quad\left(\partial_{x} \phi\right)_{R} \geq 0
$$

Then, as soon as $\Delta t>0$ is small enough, there exists $\lambda_{L}<0$ and $\lambda_{R}>0$ such that $\bar{\phi} \geq 0$.

Let us underline that the condition (2.30) to be put on both left and right variation of $\phi$ is very natural. It will be detailed in the next section when deriving a precise discrete formula for $\left(\partial_{x} \phi\right)_{L}$ and $\left(\partial_{x} \phi\right)_{R}$.

Proof. Since $\mathcal{E}_{0}$ is independent of both $\lambda_{L}$ and $\lambda_{R}$, we directly obtain the required positiveness of $\bar{\phi}$ as long as $\phi_{L}$ or $\phi_{R}$ is non zero, by imposing large enough $\left|\lambda_{L}\right|$ and $\lambda_{R}$.

Next, let us assume $\phi_{L}=\phi_{R}=0$, then we have to prove that $\mathcal{E}_{0}$ stays non negative. We have

$$
\begin{aligned}
\left.\mathcal{E}_{0}\right|_{\left\{\phi_{L}=\phi_{R}=0\right\}}= & \left(\frac{1-\mathrm{e}^{-b \Delta t}}{b \Delta t}\right)\left(D\left(\left(\partial_{x} \phi\right)_{R}-\left(\partial_{x} \phi\right)_{L}\right)+\frac{a \Delta x}{2}\left(\rho_{L}+\rho_{R}\right)\right) \\
& +\frac{a}{b}\left(\frac{1-\mathrm{e}^{-b \Delta t}}{b \Delta t}-1\right)\left(\rho_{R} u_{R}-\rho_{L} u_{L}\right) .
\end{aligned}
$$

In vacuum regions, where $\rho_{L}=\rho_{R}=0$, we get

$$
\left.\mathcal{E}_{0}\right|_{\left\{\phi_{L}=\phi_{R}=0\right\}}=D\left(\frac{1-\mathrm{e}^{-b \Delta t}}{b \Delta t}\right)\left(\left(\partial_{x} \phi\right)_{R}-\left(\partial_{x} \phi\right)_{L}\right) \geq 0 .
$$

Next, for non empty domain, we have

$$
D\left(\left(\partial_{x} \phi\right)_{R}-\left(\partial_{x} \phi\right)_{L}\right)+\frac{a \Delta x}{2}\left(\rho_{L}+\rho_{R}\right)>0 \quad \text { and } \quad \lim _{\Delta t \rightarrow 0} \frac{1-\mathrm{e}^{-b \Delta t}}{b \Delta t}=1
$$

to deduce $\mathcal{E}_{0}>0$ as soon as $\Delta t$ is small enough. The proof is thus achieved.

Arguing the non negativeness of $\bar{\phi}$, since $\phi_{L}^{\star}$ and $\phi_{R}^{\star}$ satisfy (2.15), by considering $\phi_{L}^{\star} \geq 0$ and $\phi_{R}^{\star} \geq 0$, we have

$$
0 \leq \phi_{L}^{\star} \leq \frac{\lambda_{R}-\lambda_{L}}{-\lambda_{L}} \bar{\phi} \quad \text { and } \quad 0 \leq \phi_{R}^{\star} \leq \frac{\lambda_{R}-\lambda_{L}}{\lambda_{R}} \bar{\phi}
$$


We thus propose the following modification of the intermediate concentration of chemoattractant:

$$
\begin{aligned}
\phi_{L}^{\star \star} & =\min \left(\max \left(0, \phi_{L}^{\star}\right), \frac{\lambda_{R}-\lambda_{L}}{-\lambda_{L}} \bar{\phi}\right), \\
\phi_{R}^{\star \star} & =\min \left(\max \left(0, \phi_{R}^{\star}\right), \frac{\lambda_{R}-\lambda_{L}}{\lambda_{R}} \bar{\phi}\right),
\end{aligned}
$$

where $\bar{\phi} \geq 0$ is defined by $(2.29)$.

To summarize the present section, we have derived an approximate Riemann solver in the following form:

$$
\tilde{w}\left(x / t ; w_{L}, w_{R}\right)=\left\{\begin{array}{lc}
w_{L}={ }^{t}\left(\rho_{L}, \rho_{L} u_{L}, \phi_{L}\right) & \text { if } x<\lambda_{L} t, \\
w_{L}^{\star}={ }^{t}\left(\rho_{L}^{\star \star}, q^{\star}, \phi_{L}^{\star \star}\right) & \text { if } \lambda_{L} t<x<0, \\
w_{R}^{\star}={ }^{t}\left(\rho_{R}^{\star \star}, q^{\star}, \phi_{R}^{\star \star}\right) & \text { if } 0<x<\lambda_{R} t, \\
w_{R}={ }^{t}\left(\rho_{R}, \rho_{R} u_{R}, \phi_{R}\right) & \text { if } x>\lambda_{R} t,
\end{array}\right.
$$

where the intermediate states are defined by (2.24), (2.28) and (2.31).

Once again, let us underline that this approximate Riemann solver is not fully determined since $\left(\partial_{x} \phi\right)_{L}$ and $\left(\partial_{x} \phi\right)_{R}$ do not yet admit precise definition. These two quantities will be defined in the next section according to properties to be satisfied by the designed scheme.

To conclude this section, we establish the main properties satisfied by the obtained approximate Riemann solver.

THEOREM 2.4. Let $w_{L}$ and $w_{R}$ be two constant states given in $\Omega$, where $\Omega$ is defined by (1.2). Consider the approximate Riemann solver $\tilde{w}\left(x / t ; w_{L}, w_{R}\right)$ defined by (2.32). Assume that the definition of $\left(\partial_{x} \phi\right)_{L}$ and $\left(\partial_{x} \phi\right)_{R}$ satisfies (2.30). Moreover, assume $\Delta t>0$ small enough and $\lambda_{L}<0$ and $\lambda_{R}>0$ such that both $\rho^{\mathrm{HLL}}$, defined by (2.26), and $\bar{\phi}$, defined by (2.29), are non negative. Then we have the two following properties:

(i) $\tilde{w}\left(x / t ; w_{L}, w_{R}\right)$ belongs to $\Omega$ for all $t>0$ and $x \in \mathbb{R}$.

(ii) Assume that $w_{L}$ and $w_{R}$ satisfy, in addition, the steady state condition (1.4a):

$$
u_{L}=u_{R}=0 \quad \text { and } \quad e\left(\rho_{L}\right)-\chi \phi_{L}=e\left(\rho_{R}\right)-\chi \phi_{R} .
$$

Then ${ }^{t}(\tilde{\rho}, \tilde{\rho u})\left(x / t ; w_{L}, w_{R}\right)$ stays at rest: for all $t>0$ we have

$$
{ }^{t}(\tilde{\rho}, \tilde{\rho u})\left(x / t ; w_{L}, w_{R}\right)= \begin{cases}{ }^{t}\left(\rho_{L}, 0\right) & \text { if } x<0, \\ { }^{t}\left(\rho_{R}, 0\right) & \text { if } x>0 .\end{cases}
$$

Proof. Concerning the first property, since we have $\rho^{\mathrm{HLL}} \geq 0$ and $\bar{\phi} \geq 0$, the definitions of $\rho_{L, R}^{\star \star}$ and $\phi_{L, R}^{\star \star}$, given by $(2.28)$ and $(2.31)$, immediately imply $\rho_{L, R}^{\star \star} \geq 0$ and $\phi_{L, R}^{\star \star} \geq 0$. As a consequence, $\tilde{w}\left(x / t ; w_{L}, w_{R}\right)$ belongs to $\Omega$ for all $t>0$ and $x \in \mathbb{R}$.

Concerning the second property, we have to establish that $\rho_{L}^{\star \star}=\rho_{L}, \rho_{R}^{\star \star}=\rho_{R}$ and $q^{\star}=0$ as soon as $w_{L}$ and $w_{R}$ satisfy (2.33). Since $q^{\star}$ is given by $(2.24)$ and $\left\{\rho \partial_{x} \phi\right\}$ is given by (2.18), we obviously obtain $q^{\star}=0$. Next, we evaluate the intermediate density given by (2.28). By using (2.22)-(2.23) and (2.26), we easily get

$$
\rho_{L}^{\star}=\rho_{L}, \quad \rho_{R}^{\star}=\rho_{R}, \quad \text { and } \quad \rho^{\mathrm{HLL}}=\frac{\lambda_{R} \rho_{R}-\lambda_{L} \rho_{L}}{\lambda_{R}-\lambda_{L}} .
$$

As a consequence, by definition of $\rho_{L}^{\star \star}$ and $\rho_{R}^{\star \star}$, given by (2.28), we immediately deduce the expected equalities: $\rho_{L}^{\star \star}=\rho_{L}$ and $\rho_{R}^{\star \star}=\rho_{R}$. The proof is completed. 
3. The Godunov-type scheme. Equipped with this approximate Riemann solver $\tilde{w}$, defined by (2.32), we now derive a numerical finite volume scheme of Godunov-type. To address such an issue, we first consider a uniform mesh discretization in space and time made of cells $\left(x_{i-1 / 2}, x_{i+1 / 2}\right)$ and time intervals $\left(t^{n}, t^{n+1}\right)$. For the sake of simplicity, we adopt constant time and space increments, $\Delta t$ and $\Delta x$, so that $x_{i+1 / 2}=(i+1 / 2) \Delta x$ for all $i$ in $\mathbb{Z}$ and $t^{n}=n \Delta t$ for all $n$ in $\mathbb{N}$.

Let be given an initial datum $x \mapsto w_{0}(x)$ approximated over each cell $\left(x_{i-1 / 2}, x_{i+1 / 2}\right)$ by a constant $w_{i}^{0}$ defined by (see $\left.[32,47]\right)$ :

$$
w_{i}^{0}=\frac{1}{\Delta x} \int_{x_{i-1 / 2}}^{x_{i+1 / 2}} w_{0}(x) \mathrm{d} x, \quad \forall i \in \mathbb{Z} .
$$

Next, at time $t^{n}$, we assume known an approximation $w^{n}\left(x, t^{n}\right)$ of the solution $w\left(x, t^{n}\right)$ of the system (1.1) with an initial data $w_{0}$. This approximation is piecewise constant over each cell:

$$
w^{n}\left(x, t^{n}\right)=w_{i}^{n}, \quad \text { if } x \in\left(x_{i-1 / 2}, x_{i+1 / 2}\right)
$$

Now, we evolve in time this approximation to define the updated sequence $\left(w_{i}^{n+1}\right)_{i \in \mathbb{Z}}$. This time evolution is obtained by adopting a Godunov-type strategy. We consider the juxtaposition of the approximate Riemann solvers set at each interface $x_{i+1 / 2}$. We thus define

$$
w^{n}\left(x, t^{n}+t\right)=\tilde{w}\left(\frac{x-x_{i+1 / 2}}{t} ; w_{i}^{n}, w_{i+1}^{n}\right), \quad \forall x \in\left(x_{i}, x_{i+1}\right), \quad \forall t \in(0, \Delta t),
$$

where $\tilde{w}$ is defined by (2.32). As soon as $\Delta t$ is small enough, the successive approximate Riemann solvers do not interact. The time step is thus restricted according to the following CFL-like condition:

$$
\frac{\Delta t}{\Delta x} \max _{i \in \mathbb{Z}}\left(\left|\lambda_{i+1 / 2}^{L}\right|, \lambda_{i+1 / 2}^{R}\right) \leq \frac{1}{2}
$$

where, with clear notations, $\lambda_{i+1 / 2}^{L, R}$ denote the left and right wave velocities involved in the approximate Riemann solver stated at the interface $x_{i+1 / 2}$.

The expected updated state $w_{i}^{n+1}$ is then defined by the usual $L^{2}$-projection over piecewise constant functions as follows:

$$
\begin{aligned}
w_{i}^{n+1} & =\frac{1}{\Delta x} \int_{x_{i-1 / 2}}^{x_{i+1 / 2}} w^{n}\left(x, t^{n}+\Delta t\right) \mathrm{d} x \\
& =\frac{1}{\Delta x} \int_{0}^{\Delta x / 2} \tilde{w}\left(\frac{x-x_{i-1 / 2}}{\Delta t} ; w_{i-1}^{n}, w_{i}^{n}\right) \mathrm{d} x+\frac{1}{\Delta x} \int_{-\Delta x / 2}^{0} \tilde{w}\left(\frac{x-x_{i+1 / 2}}{\Delta t} ; w_{i}^{n}, w_{i+1}^{n}\right) \mathrm{d} x \\
& =w_{i}^{n}-\frac{\Delta t}{\Delta x}\left(\lambda_{i-1 / 2}^{R}\left(w_{i}^{n}-w_{i-1 / 2}^{\star, R}\right)-\lambda_{i+1 / 2}^{L}\left(w_{i}^{n}-w_{i+1 / 2}^{\star, L}\right)\right)
\end{aligned}
$$

where $w_{i+1 / 2}^{\star, L, R}$ denote the left and right intermediate states for the approximate Riemann solver $\tilde{w}\left(x / \Delta t ; w_{i}^{n}, w_{i+1}^{n}\right)$, according to (2.24), (2.28) and (2.31).

In fact, to achieve the full characterization of the scheme, we have to propose a suitable definition for $\left(\partial_{x} \phi\right)_{i}^{n}$. Of course, this definition must be consistent with $\partial_{x} \phi\left(x_{i}, t^{n}\right)$. Multiple choices can be suggested. The first proposition will yield to 
easy developments and will ensure the required well-balance property for positive $\phi$. However, the transition with regions of vanishing $\phi$ will not be possible by adopting this first choice to discretize $\partial_{x} \phi$. Next, we will show an admissible strategy to deal with such transitions.

The first formula to approximate $\partial_{x} \phi\left(x_{i}, t^{n}\right)$ is fixed as follows:

$$
\left(\partial_{x} \phi\right)_{i}^{n}=\frac{1}{2 \Delta x}\left(\phi_{i+1}^{n}-\phi_{i-1}^{n}\right)(1+\varepsilon(\Delta x)),
$$

where $\varepsilon(\Delta x)$ is a smooth function of $\Delta x$, consistent with zero. Then $\varepsilon(\Delta x)$ must tend to zero as $\Delta x$ goes to zero. Moreover, the assumption (2.30) can be now commented. Indeed, if $\phi_{i}^{n}=\phi_{i+1}^{n}=0$ then $\left(\partial_{x} \phi\right)_{i}^{n}=-\phi_{i-1}^{n}(1+\varepsilon(\Delta x)) / 2 \Delta x \leq 0$, while if $\phi_{i-1}^{n}=\phi_{i}^{n}=0$ we get $\left(\partial_{x} \phi\right)_{i}^{n}=\phi_{i+1}^{n}(1+\varepsilon(\Delta x)) / 2 \Delta x \geq 0$. In fact, (2.30) mimics a natural condition to be put on the derivative of $\phi$ to enforce $\phi \geq 0$.

The objective is now to exhibit a relevant function $\varepsilon$ such that the required wellbalance property is satisfied. More precisely, as soon as $\gamma=2$, the numerical scheme must exactly capture the steady states given by (1.6), or (1.7), or (1.8). First, we establish a sufficient condition to be put on $\varepsilon(\Delta x)$ to enforce such a property.

Lemma 3.1. Assume $\gamma=2$. Let $\phi_{i}^{n+1}$ be given by the numerical scheme (3.2)(3.3). Let us set $A, B$ and $K$ some given constants and $C=\frac{1}{D}\left(b-\frac{a \chi}{2 \delta}\right)$.

(i) Assume $\left(\rho_{i}^{n}, \phi_{i}^{n}\right)$ be given by

$$
\begin{aligned}
& \rho_{i}^{n}=0, \\
& \phi_{i}^{n}=A \cosh \left(x_{i} \sqrt{\frac{b}{D}}\right)+B \sinh \left(x_{i} \sqrt{\frac{b}{D}}\right)>0 .
\end{aligned}
$$

We obtain $\phi_{i}^{n+1}=\phi_{i}^{n}$, if we choose

$$
\varepsilon(\Delta x)=\frac{\Delta x^{2}}{2} \frac{\frac{b}{D}}{\cosh \left(\sqrt{\frac{b}{D}} \Delta x\right)-1}-1 .
$$

(ii) Assume $C<0$ and $\left(\rho_{i}^{n}, \phi_{i}^{n}\right)$ be given by

$$
\begin{aligned}
& 2 \delta \rho_{i}^{n}-\chi \phi_{i}^{n}=K \\
& \phi_{i}^{n}=A \cos \left(x_{i} \sqrt{|C|}\right)+B \sin \left(x_{i} \sqrt{|C|}\right)-\frac{K a \chi}{2 \delta b-a \chi}>0 .
\end{aligned}
$$

We obtain $\phi_{i}^{n+1}=\phi_{i}^{n}$, if we choose

$$
\varepsilon(\Delta x)=\frac{\Delta x^{2}}{2} \frac{C}{\cos (\sqrt{|C|} \Delta x)-1}-1 .
$$

(iii) Assume $C>0$ and $\left(\rho_{i}^{n}, \phi_{i}^{n}\right)$ be given by

$$
\begin{aligned}
& 2 \delta \rho_{i}^{n}-\chi \phi_{i}^{n}=K, \\
& \phi_{i}^{n}=A \cosh \left(x_{i} \sqrt{C}\right)+B \sinh \left(x_{i} \sqrt{C}\right)-\frac{K a \chi}{2 \delta b-a \chi}>0 .
\end{aligned}
$$

We obtain $\phi_{i}^{n+1}=\phi_{i}^{n}$, if we choose

$$
\varepsilon(\Delta x)=\frac{\Delta x^{2}}{2} \frac{C}{\cosh (\sqrt{C} \Delta x)-1}-1 .
$$


From now on, let us emphasize that, for each definition (3.5), (3.7) and (3.9), we have a function $\varepsilon(\Delta x)$ consistent with zero since

$$
\lim _{\Delta x \rightarrow 0} \varepsilon(\Delta x)=0
$$

Proof. Concerning the establishment of (3.5), to enforce $\phi_{i}^{n+1}=\phi_{i}^{n}$, it is sufficient to impose for all $i$ in $\mathbb{Z}$ :

$$
\left.\mathcal{E}_{0}\right|_{\left\{\phi_{L}=\phi_{i}^{n}, \phi_{R}=\phi_{i+1}^{n}\right\}}=0,
$$

where $\mathcal{E}_{0}$ is defined by (2.16). Indeed, from (2.25) such a condition enforces the intermediate states to be at rest, to get

$$
\left.\phi_{L}^{\star}\right|_{\left\{\phi_{L}=\phi_{i}^{n}, \phi_{R}=\phi_{i+1}^{n}\right\}}=\phi_{i}^{n}>0 \quad \text { and }\left.\quad \phi_{R}^{\star}\right|_{\left\{\phi_{L}=\phi_{i}^{n}, \phi_{R}=\phi_{i+1}^{n}\right\}}=\phi_{i+1}^{n}>0 .
$$

As a consequence, the updated chemoattractant $\phi_{i}^{n+1}$, given by (3.2)-(3.3), stays at rest and we obtain $\phi_{i}^{n+1}=\phi_{i}^{n}$.

Now, by imposing $\rho_{i}^{n}=0$, we have

$$
\left.\mathcal{E}_{0}\right|_{\left\{\phi_{L}=\phi_{i}^{n}, \phi_{R}=\phi_{i+1}^{n}\right\}}=\left(\frac{1-\mathrm{e}^{-b \Delta t}}{b \Delta t}\right)\left(D\left(\left(\partial_{x} \phi\right)_{i+1}^{n}-\left(\partial_{x} \phi\right)_{i}^{n}\right)-\frac{b \Delta x}{2}\left(\phi_{i}^{n}+\phi_{i+1}^{n}\right)\right),
$$

with $\left(\partial_{x} \phi\right)_{i}^{n}$ defined by (3.3) and $\phi_{i}^{n}$ given by (3.4). The relation (3.10) turns out to be an equation with unknown $\varepsilon(\Delta x)$. By solving this equation, we straightforwardly recover the expected definition (3.5).

Adopting the same strategy, we establish both statements (ii) and (iii) to complete the proof.

We are now able to give the main result of this section, where we state the properties satisfied by the derived numerical scheme.

THEOREM 3.2. Let $\left(w_{i}^{n}\right)_{i \in \mathbb{Z}}$ be a sequence in $\Omega$. Assume $w_{i}^{n+1}$ be given by the scheme (3.2), where $\left(\partial_{x} \phi\right)_{i}$ is given by (3.3) and the function $\varepsilon(\Delta x)$ is defined by (3.5) if $\rho_{i}^{n}=0$, or (3.7) if $\rho_{i}^{n}>0$ and $C<0$, or (3.9) if $\rho_{i}^{n}>0$ and $C>0$.

The scheme is non negative preserving:

$$
\rho_{i}^{n+1} \geq 0 \quad \text { and } \quad \phi_{i}^{n+1} \geq 0 \quad \text { for all } i \in \mathbb{Z} .
$$

Moreover, if $w_{i}^{n}$ satisfies (1.4a) for all $i \in \mathbb{Z}$ :

$$
u_{i}^{n}=0 \quad \text { and } \quad e\left(\rho_{i}^{n}\right)-\chi \phi_{i}^{n}=e\left(\rho_{i+1}^{n}\right)-\chi \phi_{i+1}^{n},
$$

then both updated density and velocity stay at rest:

$$
\rho_{i}^{n+1}=\rho_{i}^{n} \quad \text { and } \quad(\rho u)_{i}^{n+1}=0 \quad \text { for all } i \in \mathbb{Z} .
$$

In addition, with $\gamma=2$, the scheme is well-balanced. Indeed, assume the initial datum $\left(w_{i}^{0}\right)_{i \in \mathbb{Z}}$ and the function $\varepsilon(\Delta x)$ respectively be given by (3.4)-(3.5), or (3.6)(3.7), or (3.8)-(3.9), then the updated states stay at rest:

$$
w_{i}^{n+1}=w_{i}^{n} \quad \text { for all } i \in \mathbb{Z} .
$$


Before we establish this result, let us remark that the definition of $\varepsilon(\Delta x)$ can be unified by adopting some relevant convex combinations such that the definition of $\varepsilon(\Delta x)$ coincides with $(3.5),(3.7),(3.9)$ according to the constraints $(3.4),(3.6),(3.8)$.

Proof. Concerning the robustness of the scheme, since $\rho_{i}^{n+1}$ and $\phi_{i}^{n+1}$ are given by (3.2), both updated density and concentration of chemoattractant are positive sum integrals of the approximate Riemann solver (2.32). Because of (2.28) and (2.31), $\rho_{i}^{n+1}$ and $\phi_{i}^{n+1}$ are positive sum of non negative quantities. As a consequence, we immediately obtain $\rho_{i}^{n+1} \geq 0$ and $\phi_{i}^{n+1} \geq 0$.

Next, we establish the preservation of both density and velocity as soon as (3.11) is satisfied. Once again, we apply a property satisfied by the associated approximate Riemann solver. Indeed, from Theorem 2.4, since (3.11) holds, we get

$$
(\tilde{\rho}, \tilde{\rho u})\left(x / t ; w_{i-1}^{n}, w_{i}^{n}\right)= \begin{cases}\left(\rho_{i-1}^{n}, 0\right) & \text { if } x<0 \\ \left(\rho_{i}^{n}, 0\right) & \text { if } x>0\end{cases}
$$

and

$$
(\tilde{\rho}, \tilde{\rho u})\left(x / t ; w_{i}^{n}, w_{i+1}^{n}\right)= \begin{cases}\left(\rho_{i}^{n}, 0\right) & \text { if } x<0 \\ \left(\rho_{i+1}^{n}, 0\right) & \text { if } x>0\end{cases}
$$

From (3.2), we immediately obtain the required preservation (3.12).

To conclude the proof, we have to establish the well-balance property with $\gamma=2$. By induction, we have just to prove $w_{i}^{n+1}=w_{i}^{n}$ as soon as $w_{i}^{n}$ satisfies a rest condition (3.4), or (3.6), or (3.8).

First, let us assume (3.4) so that we are considering a vacuum domain. By definition of the scheme (3.2), we immediately get $\rho_{i}^{n+1}=0$. Adopting $\varepsilon(\Delta x)$ given by (3.5), from Lemma 3.1 we easily deduce that $\phi_{i}^{n+1}=\phi_{i}^{n}$.

Now, let us assume that $w_{i}^{n}$ satisfies (3.6). Then $w_{i}^{n}$ satisfy (3.11) and we have proved $\rho_{i}^{n+1}=\rho_{i}^{n}$ and $(\rho u)_{i}^{n+1}=0$. Concerning the preservation of the chemoattractant $\phi_{i}^{n+1}=\phi_{i}^{n}$, once again, the result directly comes from Lemma 3.1 where we have fixed $\varepsilon(\Delta x)$ given by (3.7) and $\phi_{i}^{n}$ given by (3.6). The last well-balance property coming from (3.8) is proved by adopting the same strategy. The proof is thus achieved. 口

4. Asymptotic preserving property. In fact, the derived numerical scheme (3.2) satisfies an additional property. Indeed, it preserves asymptotic regimes contained within the system (1.1).

First, we formally exhibit asymptotic regimes satisfied by the solutions of (1.1) for long time and dominant friction. For such a regime, the velocity remains in a neighborhood of zero. We introduce velocity, time and friction rescaling as follows:

$$
u=\nu u^{\nu}, \quad t=\frac{t^{\nu}}{\nu} \quad \text { and } \quad \alpha=\frac{\alpha^{\nu}}{\nu},
$$

where $\nu$ is a positive parameter devoted to tend to zero. The system (1.1) now reads:

$$
\left\{\begin{array}{l}
\nu \partial_{t^{\nu}} \rho^{\nu}+\nu \partial_{x}\left(\rho^{\nu} u^{\nu}\right)=0 \\
\nu^{2} \partial_{t^{\nu}}\left(\rho^{\nu} u^{\nu}\right)+\partial_{x}\left(\nu^{2} \rho^{\nu}\left(u^{\nu}\right)^{2}+p\left(\rho^{\nu}\right)\right)=\chi \rho^{\nu} \partial_{x} \phi^{\nu}-\alpha^{\nu} \rho^{\nu} u^{\nu} \\
\nu \partial_{t^{\nu}} \phi^{\nu}-D \partial_{x x} \phi^{\nu}=a \rho^{\nu}-b \phi^{\nu}
\end{array}\right.
$$


We adopt a formal Chapman-Enskog expansion (see [22] and references therein):

$$
\begin{aligned}
& \rho^{\nu}=\rho^{0}+O(\nu), \\
& \rho^{\nu} u^{\nu}=\rho^{0} u^{0}+O(\nu), \\
& \phi^{\nu}=\phi^{0}+O(\nu) .
\end{aligned}
$$

We consider the evolution of ${ }^{t}\left(\rho^{0}, \rho^{0} u^{0}, \phi^{0}\right)$, which is easily seen to be governed by

$$
\left\{\begin{array}{l}
\partial_{t^{\nu}} \rho^{0}+\partial_{x}\left(\frac{\chi}{\alpha} \rho^{0} \partial_{x} \phi^{0}-\frac{1}{\alpha} \partial_{x} p\left(\rho^{0}\right)\right)=0 \\
D \partial_{x x} \phi^{0}=b \phi^{0}-a \rho^{0} \\
\rho^{0} u^{0}=-\frac{1}{\alpha} \partial_{x} p\left(\rho^{0}\right)+\frac{\chi}{\alpha} \rho^{0} \partial_{x} \phi^{0}
\end{array}\right.
$$

Now, we want that such an asymptotic behavior is preserved by the here derived scheme (3.2). Such a property, called asymptotic preserving property, was introduced by Jin [42]. Several papers [27, 8, 12, 9, 26, 34, 38, 19, 20] concern the establishment of this property, which turns out to be delicate to be satisfied. Indeed, the natural numerical viscosity implied by the scheme, in general, does not coincide with the asymptotic viscosity regime. Specific numerical procedures are derived to address such an issue. Here, the originality of the source term discretization makes the numerical strategy naturally to imply the required asymptotic preserving property. More precisely, it comes from the integral equation (2.9) and (2.10), adopted to characterize the average of the approximate Riemann solver.

In order to exhibit the asymptotic preserving property, let us assume that the approximate solution belongs to the positive domain; namely with positive intermediate densities (2.22) and (2.23) and positive chemoattractant (2.25). Moreover, we assume that the wave velocities involved in the approximate Riemann solver are symmetric:

$$
\lambda_{R}=-\lambda_{L}=\lambda \text {. }
$$

Let us emphasize that such an assumption is required to expect a relevant asymptotic diffusion regime (for instance, see [11]).

Now, we consider the scheme (3.2) but for the rescaling (4.1):

$$
\Delta t=\frac{\Delta t^{\nu}}{\nu}, \quad \alpha=\frac{\alpha^{\nu}}{\nu} \quad \text { and } \quad u_{i}^{n}=\nu\left(u^{\nu}\right)_{i}^{n} .
$$

For the sake of simplicity in the notation, we omit the exponent $\nu$ to write $\Delta t, \alpha$ and $u_{i}^{n}$.

At this level, let us assume that $\lambda=O(1)$ independently of the rescaling $\nu$. Such an assumption turns out to be fully natural since, in practice, we have $\lambda \simeq u+\sqrt{p^{\prime}(\rho)}$.

Arguing the rescaling (4.4), we perform a formal Chapman-Enskog expansion of the approximate solution:

$$
w_{i}^{n}=\left(w^{0}\right)_{i}^{n}+O(\nu)
$$

to study the discrete formulas satisfied by $\left(\rho^{0}\right)_{i}^{n},\left(u^{0}\right)_{i}^{n}$ and $\left(\phi^{0}\right)_{i}^{n}$. 
As a first step, let us exhibit the asymptotic behavior coming from the updated formulas for the momentum $(\rho u)_{i}^{n}$. From (3.2) and (4.4), we have

$$
\begin{aligned}
& \nu(\rho u)_{i}^{n+1}=\nu(\rho u)_{i}^{n}-\nu \frac{1-\mathrm{e}^{-\alpha \Delta t / \nu^{2}}}{2 \alpha \Delta x}\left(\left(\nu^{2}\left(\rho u^{2}\right)_{i+1}^{n}+p\left(\rho_{i+1}^{n}\right)\right)-\left(\nu^{2}\left(\rho u^{2}\right)_{i-1}^{n}+p\left(\rho_{i-1}^{n}\right)\right)\right. \\
& \left.-\chi \Delta x\left(\left\{\rho \partial_{x} \phi\right\}_{i-1 / 2}^{n}+\left\{\rho \partial_{x} \phi\right\}_{i+1 / 2}^{n}\right)+\frac{\alpha \Delta x}{2}\left((\rho u)_{i+1}^{n}+2(\rho u)_{i}^{n}+(\rho u)_{i-1}^{n}\right)\right) \\
& +\frac{\Delta t}{\Delta x} \frac{\lambda}{2}\left((\rho u)_{i+1}^{n}-2(\rho u)_{i}^{n}+(\rho u)_{i-1}^{n}\right) .
\end{aligned}
$$

Let us remark that the CFL restriction (3.1) now reads

$$
\frac{\Delta t}{\nu \Delta x} \max _{i \in \mathbb{Z}}\left(\left|\lambda_{i+1 / 2}^{L}\right|, \lambda_{i+1 / 2}^{R}\right) \leq \frac{1}{2},
$$

where we have assumed $\lambda_{i+1 / 2}^{L, R}=O(1)$, so that $\Delta t / \Delta x=O(\nu)$. Without ambiguity, we write $\Delta t=C_{C F L} \nu \Delta x$, where $C_{C F L}>0$ is a constant defined according to (3.1).

Let us now replace $\frac{\Delta t}{\Delta x}$ by $C_{C F L} \nu$ in (4.6) and simplify by $\nu$ to write

$$
\begin{aligned}
& (\rho u)_{i}^{n+1}=(\rho u)_{i}^{n}-\frac{1-\mathrm{e}^{-\alpha \Delta t / \nu^{2}}}{2 \alpha \Delta x}\left(\left(\nu^{2}\left(\rho u^{2}\right)_{i+1}^{n}+p\left(\rho_{i+1}^{n}\right)\right)-\left(\nu^{2}\left(\rho u^{2}\right)_{i-1}^{n}+p\left(\rho_{i-1}^{n}\right)\right)\right. \\
& \left.-\chi \Delta x\left(\left\{\rho \partial_{x} \phi\right\}_{i-1 / 2}^{n}+\left\{\rho \partial_{x} \phi\right\}_{i+1 / 2}^{n}\right)+\frac{\alpha \Delta x}{2}\left((\rho u)_{i+1}^{n}+2(\rho u)_{i}^{n}+(\rho u)_{i-1}^{n}\right)\right) \\
& +\frac{\lambda C_{C F L}}{2}\left((\rho u)_{i+1}^{n}-2(\rho u)_{i}^{n}+(\rho u)_{i-1}^{n}\right) .
\end{aligned}
$$

In the limit $\nu$ tends to zero, straightforward computations yield to the following relation satisfied by the first-order Chapmann-Enskog expansion of the momentum:

$$
\begin{aligned}
\left(\rho^{0} u^{0}\right)_{i}^{n+1}= & \frac{1}{\alpha}\left(-\frac{p\left(\rho_{i+1}^{n}\right)-p\left(\rho_{i-1}^{n}\right)}{2 \Delta x}+\frac{\chi}{2}\left(\left\{\rho \partial_{x} \phi\right\}_{i+1 / 2}^{n}+\left\{\rho \partial_{x} \phi\right\}_{i-1 / 2}^{n}\right)\right) \\
& +\frac{1}{2}\left(\lambda C_{C F L}-\frac{1}{2}\right)\left(\left(\rho^{0} u^{0}\right)_{i+1}^{n}-2\left(\rho^{0} u^{0}\right)_{i}^{n}+\left(\rho^{0} u^{0}\right)_{i-1}^{n}\right) .
\end{aligned}
$$

In addition, since we are considering diffusive regime with smooth solutions, then we have

$$
\begin{aligned}
&\left(\rho^{0} u^{0}\right)_{i+1}^{n}-2\left(\rho^{0} u^{0}\right)_{i}^{n}+\left(\rho^{0} u^{0}\right)_{i-1}^{n}=O\left(\Delta x^{2}\right), \\
&\left(\rho^{0} u^{0}\right)_{i}^{n+1}-\left(\rho^{0} u^{0}\right)_{i}^{n}=O(\Delta t) .
\end{aligned}
$$

As a consequence, and since (4.8) rewrites

$$
\begin{aligned}
\left(\rho^{0} u^{0}\right)_{i}^{n}= & \frac{1}{\alpha}\left(-\frac{p\left(\rho_{i+1}^{n}\right)-p\left(\rho_{i-1}^{n}\right)}{2 \Delta x}+\frac{\chi}{2}\left(\left\{\rho \partial_{x} \phi\right\}_{i+1 / 2}^{n}+\left\{\rho \partial_{x} \phi\right\}_{i-1 / 2}^{n}\right)\right) \\
& +\frac{1}{2}\left(\lambda C_{C F L}-\frac{1}{2}\right)\left(\left(\rho^{0} u^{0}\right)_{i+1}^{n}-2\left(\rho^{0} u^{0}\right)_{i}^{n}+\left(\rho^{0} u^{0}\right)_{i-1}^{n}\right)+\left(\left(\rho^{0} u^{0}\right)_{i}^{n}-\left(\rho^{0} u^{0}\right)_{i}^{n+1}\right),
\end{aligned}
$$

we notice that we recover the required consistance with (4.2). 
Now, we turn considering the updated formula for the density. By adopting the rescaling (4.4), we get

$$
\begin{aligned}
\left(\rho^{0}\right)_{i}^{n+1}= & \left(\rho^{0}\right)_{i}^{n}-\frac{\Delta t}{\Delta x} \frac{e\left(\left(\rho^{0}\right)_{i}^{n}\right)}{\left(\rho^{0}\right)_{i}^{n}}\left(\ell_{i-1 / 2}\left(\left(\rho^{0} u^{0}\right)_{i}^{n}-\left(\rho^{0} u^{0}\right)_{i-1}^{n}\right)+\ell_{i+1 / 2}\left(\left(\rho^{0} u^{0}\right)_{i+1}^{n}-\left(\rho^{0} u^{0}\right)_{i}^{n}\right)\right) \\
& +\lambda C_{C F L}\left(\ell_{i+1 / 2}\left(e\left(\left(\rho^{0}\right)_{i+1}^{n}\right)-e\left(\left(\rho^{0}\right)_{i}^{n}\right)\right)-\ell_{i-1 / 2}\left(e\left(\left(\rho^{0}\right)_{i}^{n}\right)-e\left(\left(\rho^{0}\right)_{i-1}^{n}\right)\right)\right) \\
& +\lambda C_{C F L} \chi\left(\ell_{i+1 / 2}\left(\phi_{i+1}^{n}-\phi_{i}^{n}\right)-\ell_{i-1 / 2}\left(\phi_{i}^{n}-\phi_{i-1}^{n}\right)\right),
\end{aligned}
$$

where we have set

$$
\ell_{i+1 / 2}=\frac{1}{e\left(\left(\rho^{0}\right)_{i}^{n}\right) / \rho_{i}^{n}+e\left(\left(\rho^{0}\right)_{i+1}^{n}\right) / \rho_{i+1}^{n}} .
$$

Once again, by involving smooth solutions, we have

$$
\begin{aligned}
& \ell_{i+1 / 2}\left(e\left(\left(\rho^{0}\right)_{i+1}^{n}\right)-e\left(\left(\rho^{0}\right)_{i}^{n}\right)\right)-\ell_{i+1 / 2}\left(e\left(\left(\rho^{0}\right)_{i}^{n}\right)-e\left(\left(\rho^{0}\right)_{i-1}^{n}\right)\right)=O\left(\Delta x^{2}\right), \\
& \ell_{i+1 / 2}\left(\phi_{i+1}^{n}-\phi_{i}^{n}\right)-\ell_{i-1 / 2}\left(\phi_{i}^{n}-\phi_{i-1}^{n}\right)=O(\Delta x) .
\end{aligned}
$$

Plugging (4.8) into (4.9), we easily see that the relation (4.9) is consistent with the evolution diffusive law for $\rho$ given in (4.2).

Finally, concerning the rescaled formula to update the concentration of chemoattractant, straightforward calculations give

$$
\begin{aligned}
\left(\phi^{0}\right)_{i}^{n+1}= & \left(\phi^{0}\right)_{i}^{n}+\frac{D}{b} \frac{\left(\partial_{x} \phi\right)_{i+1}^{n}-\left(\partial_{x} \phi\right)_{i-1}^{n}}{2 \Delta x}-\frac{\left(\phi^{0}\right)_{i-1}^{n}+2\left(\phi^{0}\right)_{i}^{n}+\left(\phi^{0}\right)_{i+1}^{n}}{4} \\
& +\frac{a}{b} \frac{\left(\rho^{0}\right)_{i-1}^{n}+2\left(\rho^{0}\right)_{i}^{n}+\left(\rho^{0}\right)_{i+1}^{n}}{4}
\end{aligned}
$$

which is consistent to the evolution law for $\phi$ given by (4.2).

As a consequence, we have proved that the scheme (3.2) is asymptotic preserving.

5. Numerical results. We present in this section some numerical results. First, we study the behaviour of the model when perturbing a stationary solution. Then, numerous simulations are performed in order to look at the influence of parameters on the stationary state for a given (non stationary) initial solution.

First, we are interested in the behaviour of our scheme on equilibrium solutions. We consider $\gamma=2$ and the following exact equilibrium solution (see [51]):

$$
\begin{gathered}
\phi(x)=\left\{\begin{array}{lc}
\frac{2 \delta b K}{\tau \chi D} \frac{\cos (\sqrt{\tau} x)}{\cos (\sqrt{\tau} \bar{x})}-\frac{a K}{\tau D}, & \text { for } x \in[0, \bar{x}], \\
-\frac{2 \delta K}{\chi} \frac{\cosh \left(\sqrt{\frac{b}{D}}(x-L)\right)}{\cosh \left(\sqrt{\frac{b}{D}}(\bar{x}-L)\right)}, & \text { for } x \in(\bar{x}, L],
\end{array}\right. \\
\rho(x)= \begin{cases}\frac{\chi}{2 \delta} \phi(x)+\frac{D}{b} \frac{M \tau^{3 / 2}}{\tan (\sqrt{\tau} \bar{x})-\sqrt{\tau} \bar{x}}, & \text { for } x \in[0, \bar{x}], \\
0, & \text { for } x \in(\bar{x}, L],\end{cases} \\
u(x)=0,
\end{gathered}
$$

where $\tau=\frac{1}{D}\left(\frac{a \chi}{2 \delta}-b\right)$ and $\bar{x}$ solution of $\sqrt{\frac{b}{\tau D}} \tan (\sqrt{\tau} \bar{x})=\tanh \left(\sqrt{\frac{b}{D}}(\bar{x}-L)\right)$. 

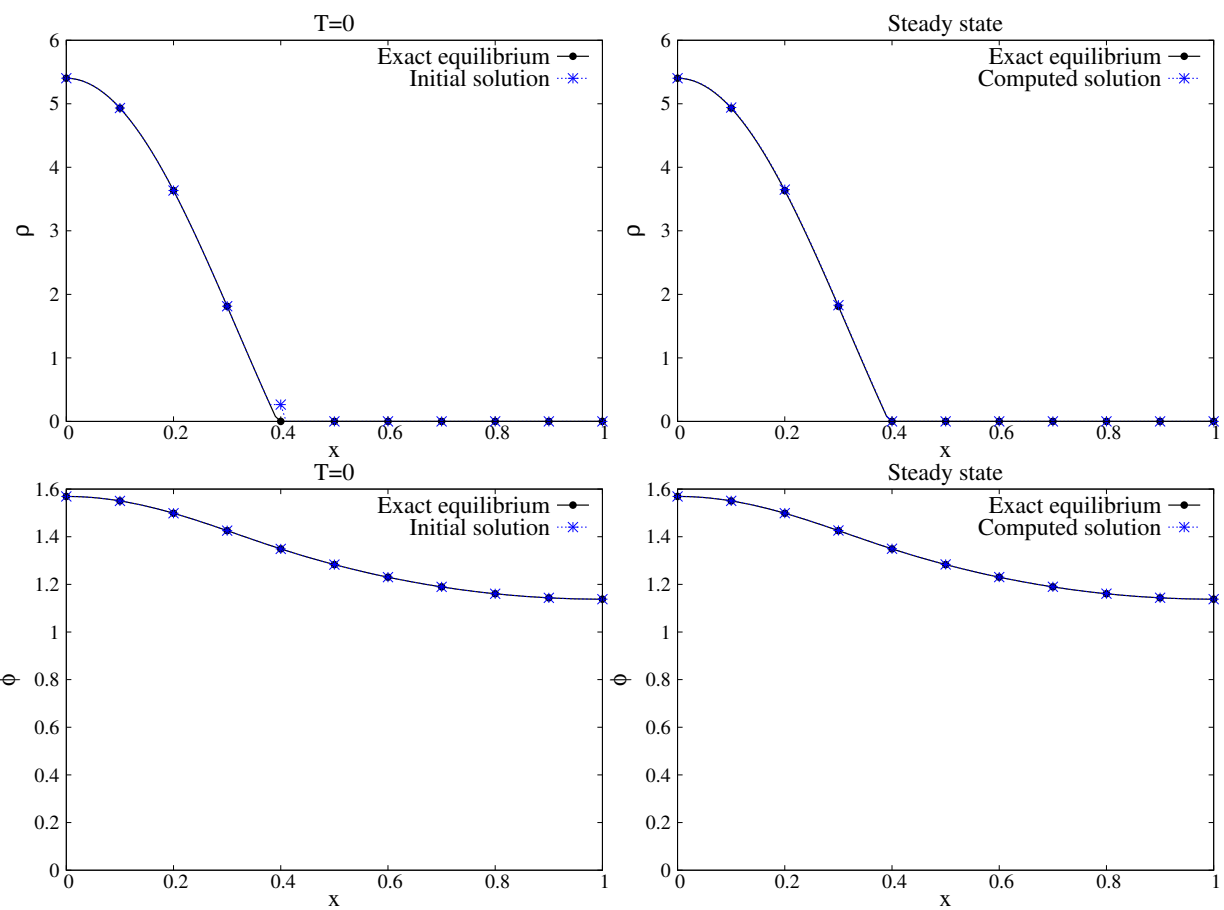

FIGURE 5.1. $\rho$ (on the top) and $\phi$ (on the bottom) as a function of $x$ at time $t=0$ (left) and $t=T_{\text {stat }}$ (right). Computed solutions compared to exact equilibrium given by (5.1)-(5.2)-(5.3).

When considering (5.1)-(5.2)-(5.3) as initial solution, we verify that the presented scheme preserves this steady state. Here the domain of computation is bounded. We have adopted Neumann boundary conditions for the density $\rho$ and the chemoattractant $\phi$, while Dirichlet conditions have been imposed for the velocity with $u(0, t)=$ $u(L, t)=0$.

Concerning the choice of the velocities involved within the approximate Riemann solver, they have been fixed as follows:

$$
\lambda_{R}=-\lambda_{L}=\lambda
$$

where

$$
\lambda=\max \left(\left|u_{L} \pm \sqrt{\varepsilon \gamma \rho_{L}^{\gamma-1}}\right|,\left|u_{R} \pm \sqrt{\varepsilon \gamma \rho_{R}^{\gamma-1}}\right|\right)
$$

From a numerical point of view, such a choice of $\lambda_{L}$ and $\lambda_{R}$ has satisfied the main assumption of Theorem 2.4 .

Moreover, if we slightly perturb this steady state, we observe that the solution goes back to the equilibrium (5.1)-(5.2)-(5.3). On the left of Figure 5.1, we present the perturbed density $\rho$ (on the top) and the concentration of chemoattractant $\phi$ (on the bottom) given by (5.1) at time $T=0$. On the right of this figure, we present these quantities at equilibrium. Parameters have the following values: $a=b=D=\delta=1$, $\gamma=2, \chi=50, L=1, \Delta x=0.01$ and solutions are compared to the exact equilibrium given by (5.1)-(5.2)-(5.3).

Next, we are interested in the different kinds of stationary solutions we can obtain, varying some parameters. More precisely, we study test cases proposed by Natalini, 

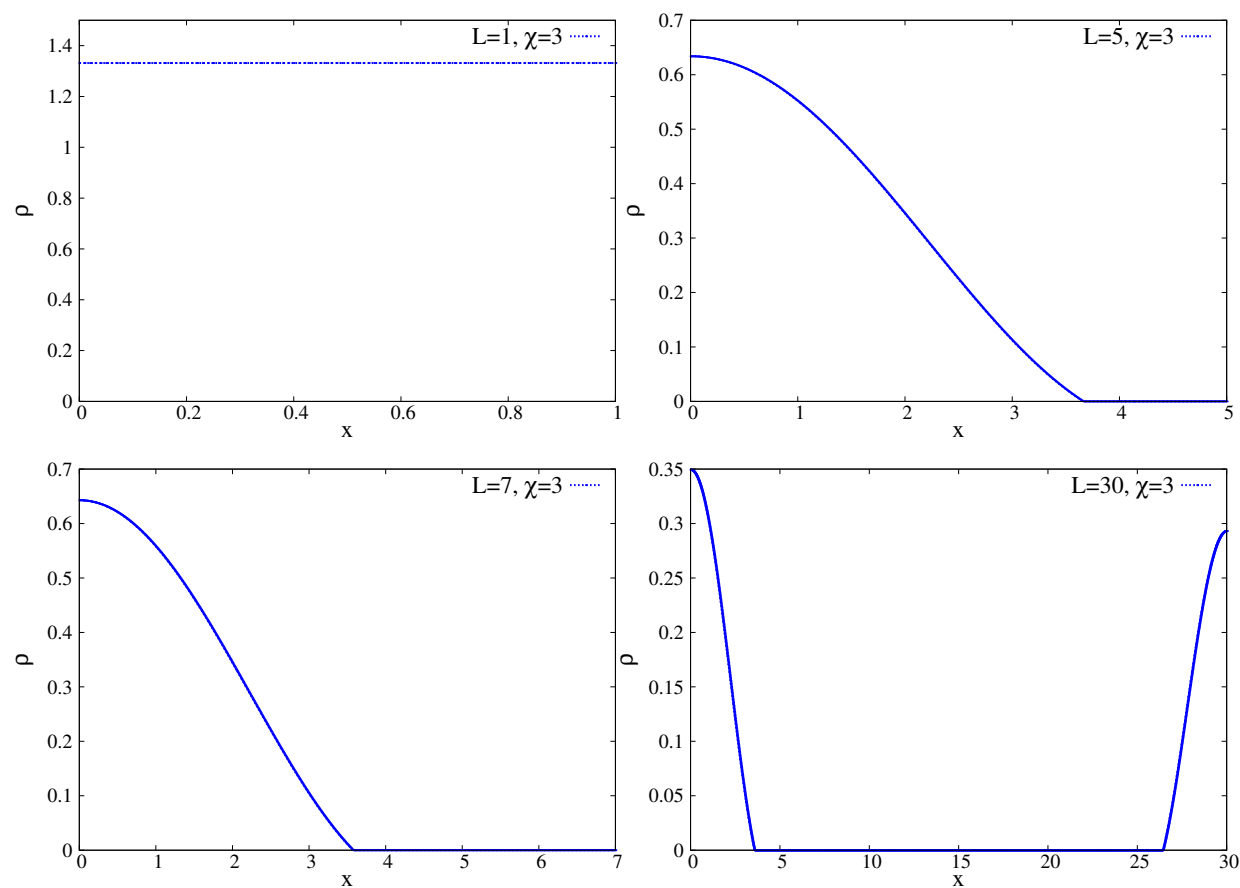

Figure 5.2. $\rho\left(x, t=T_{\text {stat }}\right)$ for $L=1$ (top left), $L=5$ (top right), $L=7$ (bottom left) and $L=30$ (bottom right).

\begin{tabular}{|c|c|c|}
\hline $\mathrm{Nb}$ of cells & Error in $L^{1}=L^{2}=L^{\infty}$-norm & Order \\
\hline 50 & $2.69 \mathrm{E}-2$ & - \\
100 & $1.33 \mathrm{E}-2$ & 1.358 \\
200 & $6.62 \mathrm{E}-3$ & 1.340 \\
400 & $3.30 \mathrm{E}-3$ & 1.328 \\
800 & $1.62 \mathrm{E}-3$ & 1.344 \\
1600 & $6.60 \mathrm{E}-4$ & 1.536 \\
\hline
\end{tabular}

Order of convergence in the test $L=1$ given in $L^{1}, L^{2}$ and $L^{\infty}$-norm.

Ribot and Twarogowska in [51] (see also [56]). The quantities $\rho, u$ and $\phi$ are initially given by $\rho(x, 0)=\xi\left(1+\sin \left(4 \pi\left|x-\frac{L}{4}\right|\right)\right), u(x, 0)=0$ and $\phi(x, 0)=0$, where $\xi$ is a constant such that $\int_{0}^{L_{x}} \rho(x, 0) \mathrm{d} x=M_{0}$ with $M_{0}=1.3183$ the total mass. We look at the influence of the following parameters: $L, \chi$ and $\gamma$ on the stationary state.

We first take $a=b=D=\delta=1, \gamma=2, \chi=3, \Delta x=0.01$. We represent in Figure 5.2, respectively Figure 5.3, the density $\rho$, respectively the momentum $\rho u$, as a function of $x$ at time $T_{\text {stat }}$ such that $\left\|\rho\left(x, T_{\text {stat }}\right)-\rho\left(x, T_{\text {stat }}-\Delta t\right)\right\|_{\infty}<5 \times 10^{-12}$, for different values of $L$. In Figure 5.4, we display the residues during time of $\rho$ and $\rho u$, which confirms that we have reached a steady state. We recover the same steady states as in [51] except for $L=30$. The figure corresponding to this test with $L=30$ in [51] (Figure 4.7 page 34) makes us suppose that they have not reach the steady state, perhaps because of the computational time. Since the exact solution is known in the test involving $L=1(\rho=1.3183, \phi=1.3183, u=0)$, we have evaluated the order of the scheme given in Table 5.1. 

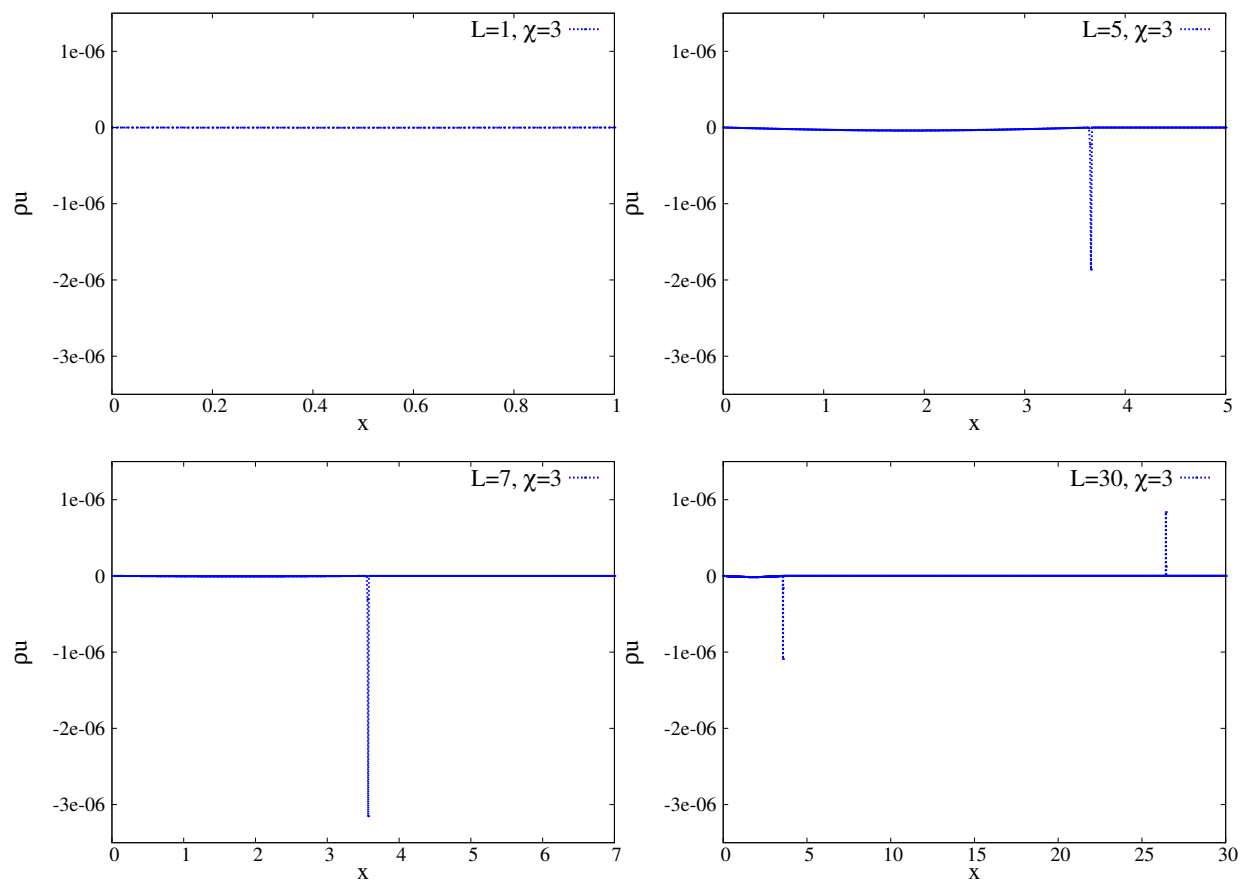

Figure 5.3. $\rho u\left(x, t=T_{\text {stat }}\right)$ for $L=1$ (top left), $L=5$ (top right), $L=7$ (bottom left) and $L=30$ (bottom right).
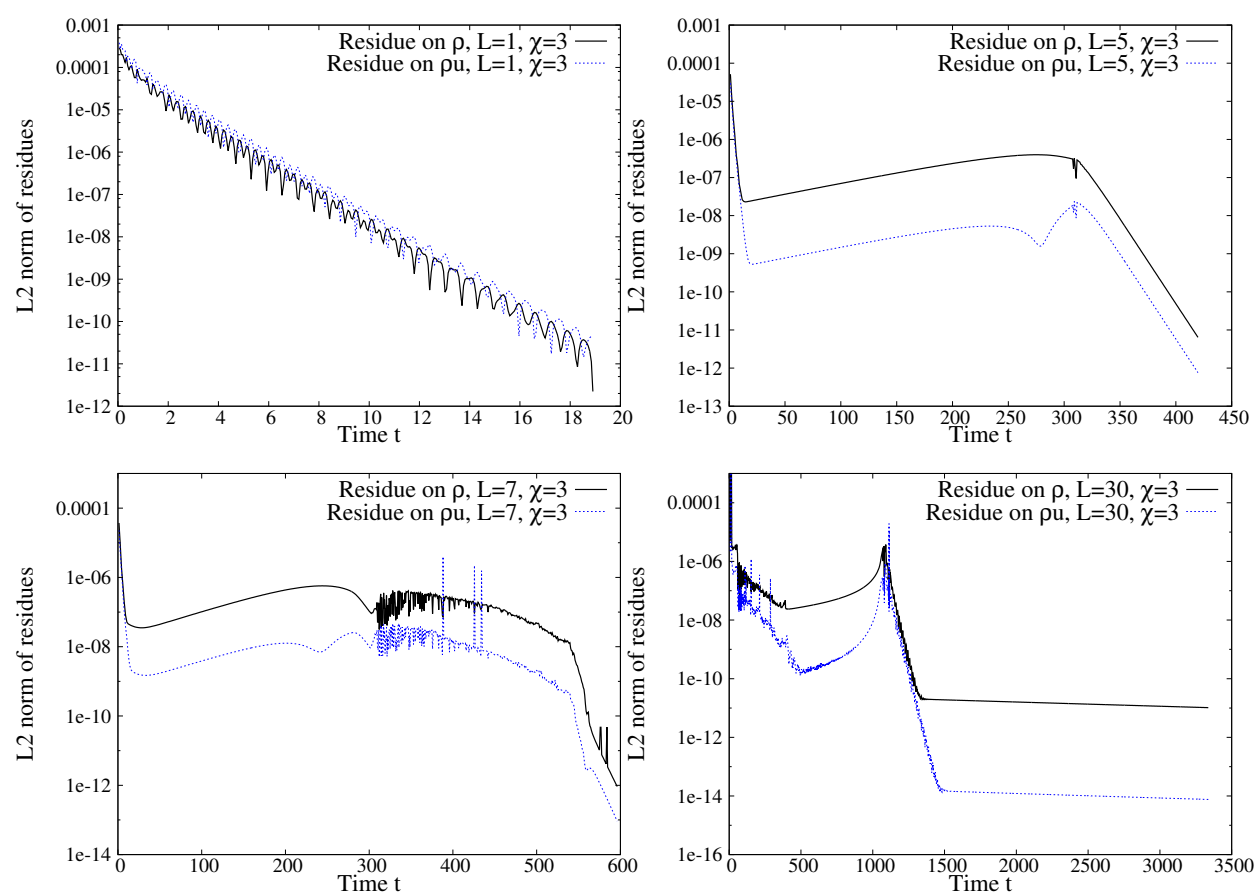

Figure 5.4. Evolution of residues on $\rho$ and $\rho u$ in $L^{2}$-norm as a function of time for $L=1$ (top left), $L=5$ (top right), $L=7$ (bottom left) and $L=30$ (bottom right). 

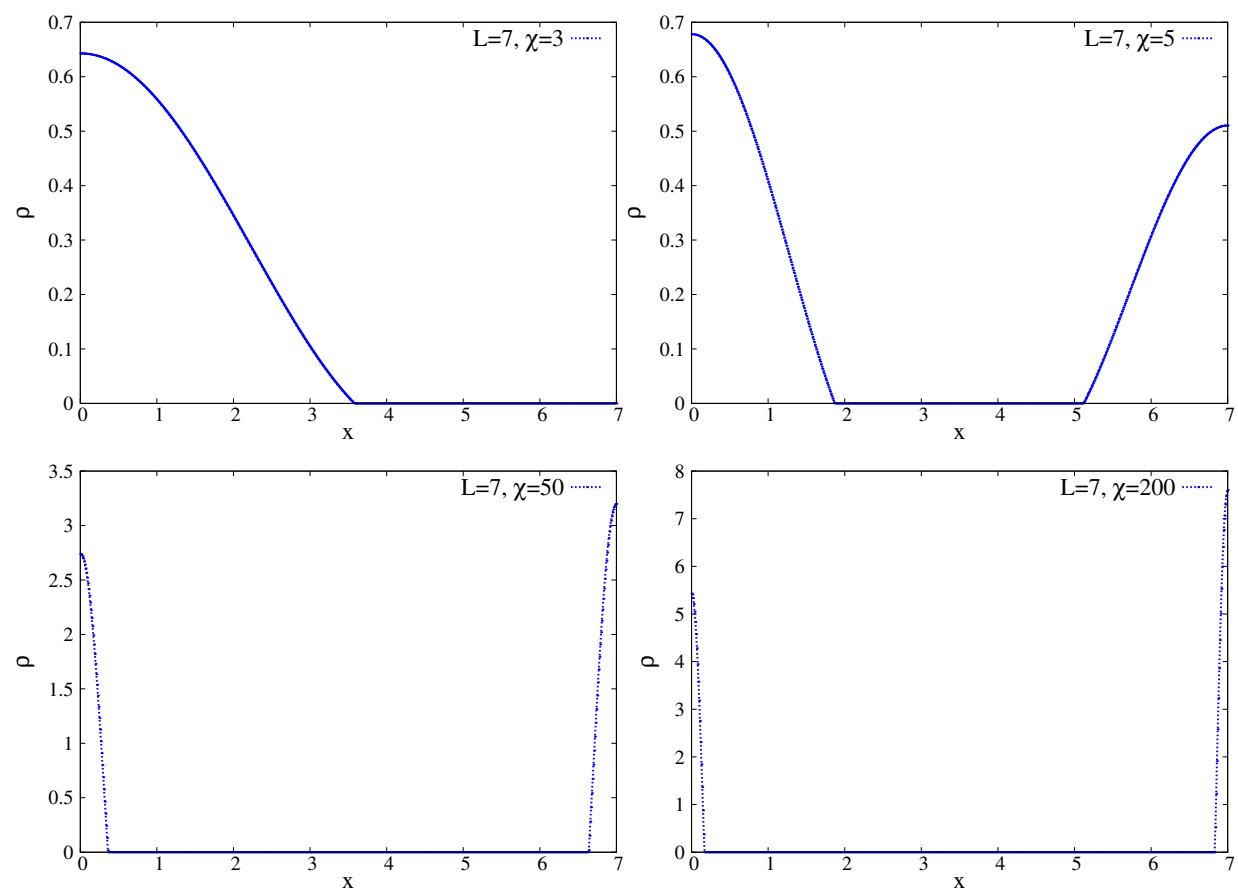

Figure 5.5. $\rho\left(x, t=T_{\text {stat }}\right)$ for $\chi=3$ (top left), $\chi=5$ (top right), $\chi=50$ (bottom left) and $\chi=200$ (bottom right).

We now fix $L=7$ and represent, in Figure 5.5, $\rho$ as a function of $x$ at time $T_{\text {stat }}$ for varying $\chi$. The obtained numerical results are in a good agreement with the simulations presented in [51].

Finally, we present in Figure 5.6 the influence of $\gamma$, with the following parameters: $a=20, b=10, D=0.1, \delta=1, \chi=10, L=3, \Delta x=0.01$ and $\xi=1$. For these tests, we also recover the results of [51].

6. Conclusions. The Finite Volume scheme presented in this paper has been constructed by deriving a pertinent approximate Riemann solver, in order to exactly preserve equilibria of particular interest for the 1D chemotaxis model (1.1). As in [51], the scheme is well-balanced in the two first variables $\rho$ and $\rho u$ when $\gamma>1$. But here, steady states at rest are also exactly preserved in the third variable $\phi$ in the case $\gamma=2$. The key point is to use the analytic expression of equilibria when defining a discrete approximation of $\partial_{x} \phi$ on the mesh. Moreover, the designed scheme is able to deal with vacuum regions and is Asymptotic Preserving when the friction term is dominant. Many numerical results have been performed for different initial datas and parameters in order to validate the scheme. As expected, results are similar to those of [51]. Some of them are presented in this paper for illustrating the capture of equilibrium solutions and their diversity, depending on the domain length $L$, the constants $\chi$ and $\gamma$.

Concerning a practical point of view, this method must be now extended to consider accurate 2D simulations. The order of approximation can be extended by adopting usual technique (MUSCL approach for instance). The main difficulty coming from high-order extensions is to preserve the steady states. Concerning both density and momentum high-order reconstruction, it is possible to adopt reconstruction based 

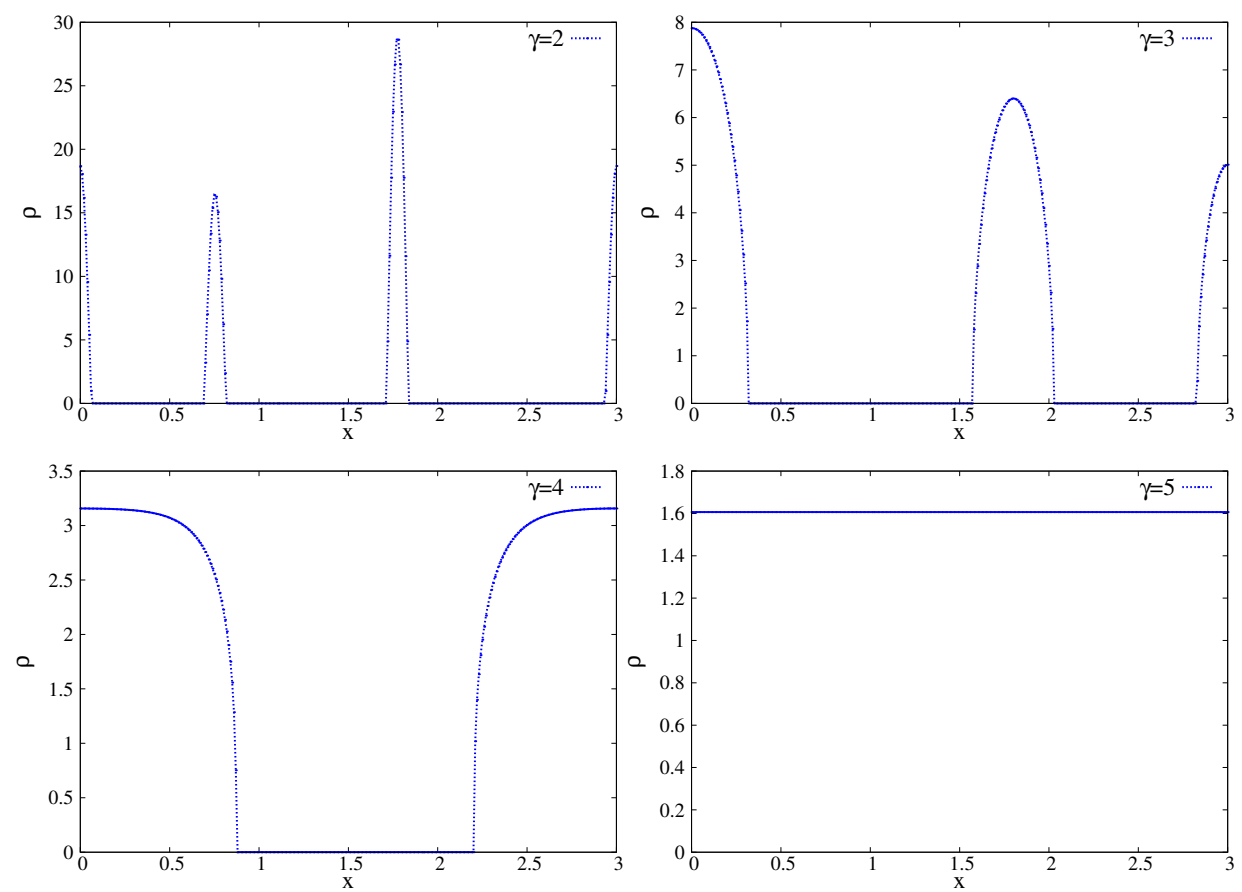

Figure 5.6. $\rho\left(x, t=T_{\text {stat }}\right)$ for $\gamma=2$ (top left), $\gamma=3$ (top right), $\gamma=4$ (bottom left) and $\gamma=5$ (bottom right).

on the steady states definition (1.4a). The reconstruction of the chemoattractant concentration turns out to be more severe since we have to preserve nonlinear functions given by (1.6) or (1.7) or (1.8). Next, to get 2D extensions, some usual techniques can be considered (for instance, see [10] and references therein). In fact, the problem stays in the description of $2 \mathrm{D}$ steady states at rest.

Acknowledgements. The authors thank the project ANR-12-IS01-0004 GeoNum to partially support this work.

\section{REFERENCES}

[1] E. Audusse, F. Bouchut, M.-O. Bristeau, R. Klein, and B. Perthame. A fast and stable wellbalanced scheme with hydrostatic reconstruction for shallow water flows. SIAM Journal on Scientific Computing, 25(6):2050-2065, 2004.

[2] E. Audusse and M.-O. Bristeau. A well-balanced positivity preserving second-order scheme for shallow water flows on unstructured meshes. Journal of Computational Physics, 206(1):311-333, 2005.

[3] E. Audusse, C. Chalons, and P. Ung. A very simple well-balanced positive and entropysatisfying scheme for the shallow-water equations. Communications in Mathematical Sciences, 13(5):1317-1332, 2015.

[4] M. Ben-Artzi and J. Falcovitz. A second-order Godunov-type scheme for compressible fluid dynamics. J. Comput. Phys., 55(1):1-32, 1984.

[5] M. Ben-Artzi and J. Li. Hyperbolic balance laws: Riemann invariants and the generalized Riemann problem. Numerische Mathematik, 106(3):369-425, 2007.

[6] M. Ben-Artzi, J. Li, and G. Warnecke. A direct Eulerian GRP scheme for compressible fluid flows. Journal of Computational Physics, 218(1):19-43, 2006.

[7] C. Berthon, M. Breuß, and M.-O. Titeux. A relaxation scheme for the approximation of the pressureless Euler equations. Numerical Methods for Partial Differential Equations, $22(2): 484-505,2006$. 
[8] C. Berthon, C. Chalons, and R. Turpault. Asymptotic-preserving godunov-type numerical schemes for hyperbolic systems with stiff and nonstiff relaxation terms. Numerical Methods for Partial Differential Equations, 29:1149-1172, 2013.

[9] C. Berthon, P. Charrier, and B. Dubroca. An hllc scheme to solve the $\mathrm{m} 1 \mathrm{model}$ of radiative transfer in two space dimensions. Journal of Scientific Computing, 31(3):347-389, 2007.

[10] C. Berthon and F. Foucher. Efficient well-balanced hydrostatic upwind schemes for shallowwater equations. Journal of Computational Physics, 231(15):4993-5015, 2012.

[11] C. Berthon, P. G. LeFloch, and R. Turpault. Late-time/stiff-relaxation asymptotic-preserving approximations of hyperbolic equations. Math. Comp., 82:831-860, 2013.

[12] C. Berthon and R. Turpault. Asymptotic preserving hll schemes. Numerical methods for partial differential equations, 27(6):1396-1422, 2011.

[13] F. Bouchut. Nonlinear stability of finite volume methods for hyperbolic conservation laws and well-balanced schemes for sources. Frontiers in Mathematics. Birkhäuser Verlag, Basel, 2004.

[14] F. Bouchut and F. James. Duality solutions for pressureless gases, monotone scalar conservation laws, and uniqueness. Communications in partial differential equations, 24(11-12):21732189, 1999.

[15] F. Bouchut, S. Jin, and X. Li. Numerical approximations of pressureless and isothermal gas dynamics. SIAM Journal on Numerical Analysis, 41(1):135-158, 2003.

[16] F. Bouchut, C. Klingenberg, and K. Waagan. A multiwave approximate Riemann solver for ideal MHD based on relaxation II: numerical implementation with 3 and 5 waves. Numer. Math., 115(4):647-679, 2010.

[17] F. Bouchut, H. Ounaissa, and B. Perthame. Upwinding of the source term at interfaces for Euler equations with high friction. Computers \& Mathematics with Applications, 53(3):361-375, 2007.

[18] S. Bryson, Y. Epshteyn, A. Kurganov, and G. Petrova. Well-balanced positivity preserving central-upwind scheme on triangular grids for the Saint-Venant system. ESAIM Math. Model. Numer. Anal., 45(3):423-446, 2011.

[19] C. Buet and S. Cordier. An asymptotic preserving scheme for hydrodynamics radiative transfer models. Numerische Mathematik, 108(2):199-221, 2007.

[20] C. Buet and B. Despres. Asymptotic preserving and positive schemes for radiation hydrodynamics. Journal of Computational Physics, 215(2):717-740, 2006.

[21] M. J. Castro, J. A. García-Rodríguez, J. M. González-Vida, and C. Parés. Solving shallowwater systems in 2D domains using finite volume methods and multimedia sse instructions. Journal of Computational and Applied Mathematics, 221(1):16-32, 2008.

[22] C. Cercignani. The Boltzmann equation and its applications. Applied Mathematical Sciences, 67, Springer-Verlag, New York, 1988.

[23] C. Chalons, F. Coquel, E. Godlewski, P.-A. Raviart, and N. Seguin. Godunov-type schemes for hyperbolic systems with parameter-dependent source. The case of Euler system with friction. Math. Models Methods Appl. Sci., 20(11):2109-2166, 2010.

[24] S. Chen, D. Huang, and X. Han. The generalized Riemann problem for first order quasilinear hyperbolic systems of conservation laws I. Bull. Korean Math. Soc, 46(3):409-434, 2009.

[25] A. Chertock, M. Dudzinski, A. Kurganov, and M. Lukáčová-Medvidová. Well-balanced schemes for the shallow water equations with coriolis forces. Submitted.

[26] F. Filbet and S. Jin. A class of asymptotic-preserving schemes for kinetic equations and related problems with stiff sources. Journal of Computational Physics, 229(20):7625-7648, 2010.

[27] F. Filbet and A. Rambaud. Analysis of an asymptotic preserving scheme for relaxation systems. ESAIM Math. Model. Numer. Anal., 47(2):609-633, 2013.

[28] F. Filbet and C.-W. Shu. Approximation of hyperbolic models for chemosensitive movement. SIAM J. Sci. Comput., 27(3):850-872, 2005.

[29] G. Gallice. Solveurs simples positifs et entropiques pour les systèmes hyperboliques avec terme source. C. R. Math. Acad. Sci. Paris, 334(8):713-716, 2002.

[30] G. Gallice. Positive and entropy stable Godunov-type schemes for gas dynamics and MHD equations in Lagrangian or Eulerian coordinates. Numer. Math., 94(4):673-713, 2003.

[31] A Gamba, D Ambrosi, A Coniglio, A De Candia, S Di Talia, E Giraudo, G Serini, Luigi Preziosi, and Federico Bussolino. Percolation, morphogenesis, and burgers dynamics in blood vessels formation. Physical review letters, 90(11):118101(1-4), 2003.

[32] E. Godlewski and P.-A. Raviart. Numerical approximation of hyperbolic systems of conservation laws, volume 118 of Applied Mathematical Sciences. Springer-Verlag, New York, 1996.

[33] L. Gosse. A well-balanced flux-vector splitting scheme designed for hyperbolic systems of conservation laws with source terms. Computers $\&$ Mathematics with Applications, 39(9):135- 
$159,2000$.

[34] L. Gosse. Asymptotic-preserving and well-balanced schemes for the 1D Cattaneo model of chemotaxis movement in both hyperbolic and diffusive regimes. J. Math. Anal. Appl., 388(2):964-983, 2012.

[35] L. Gosse. Maxwellian decay for well-balanced approximations of a super-characteristic chemotaxis model. SIAM Journal on Scientific Computing, 34(1):A520-A545, 2012.

[36] L. Gosse and G. Toscani. An asymptotic-preserving well-balanced scheme for the hyperbolic heat equations. Comptes Rendus Mathematique, 334(4):337-342, 2002.

[37] L. Gosse and G. Toscani. Space localization and well-balanced schemes for discrete kinetic models in diffusive regimes. SIAM journal on numerical analysis, 41(2):641-658, 2003.

[38] L. Gosse and G. Toscani. Asymptotic-preserving \& well-balanced schemes for radiative transfer and the rosseland approximation. Numerische Mathematik, 98(2):223-250, 2004.

[39] J. M Greenberg and A.-Y. Leroux. A well-balanced scheme for the numerical processing of source terms in hyperbolic equations. SIAM Journal on Numerical Analysis, 33(1):1-16, 1996.

[40] F. Guarguaglini, C. Mascia, R. Natalini, and M. Ribot. Stability of constant states and qualitative behavior of solutions to a one dimensional hyperbolic model of chemotaxis. Discrete Contin. Dyn. Syst. Ser. B, 12:39-76, 2009.

[41] A. Harten, P.D. Lax, and B. Van Leer. On upstream differencing and Godunov-type schemes for hyperbolic conservation laws. SIAM review, 25:35-61, 1983.

[42] S. Jin. Efficient asymptotic-preserving (ap) schemes for some multiscale kinetic equations. SIAM Journal on Scientific Computing, 21(2):441-454, 1999.

[43] S. Jin. A steady-state capturing method for hyperbolic systems with geometrical source terms. ESAIM: Mathematical Modelling and Numerical Analysis, 35(04):631-645, 2001.

[44] D. Lannes and F. Marche. A new class of fully nonlinear and weakly dispersive Green-Naghdi models for efficient 2D simulations. J. Comput. Phys., 282:238-268, 2015.

[45] P. G. LeFloch. Shock waves for nonlinear hyperbolic systems in nonconservative form. Institute for Math. and its Appl., Minneapolis, 593:1989, 1989.

[46] P. G. LeFloch and M. D. Thanh. A godunov-type method for the shallow water equations with discontinuous topography in the resonant regime. Journal of Computational Physics, 230(20):7631-7660, 2011.

[47] R. J. LeVeque. Finite volume methods for hyperbolic problems. Cambridge Texts in Applied Mathematics. Cambridge University Press, Cambridge, 2002.

[48] R. J. LeVeque and M. Pelanti. A class of approximate Riemann solvers and their relation to relaxation schemes. Journal of Computational Physics, 172(2):572-591, 2001.

[49] Q. Liang and F. Marche. Numerical resolution of well-balanced shallow water equations with complex source terms. Advances in water resources, 32(6):873-884, 2009.

[50] R. Natalini and M. Ribot. Asymptotic high order mass-preserving schemes for a hyperbolic model of chemotaxis. SIAM J. Numer. Anal., 50(2):883-905, 2012.

[51] R. Natalini, M. Ribot, and M. Twarogowska. A well-balanced numerical scheme for a one dimensional quasilinear hyperbolic model of chemotaxis. Commun. Math. Sci., 12(1):13$39,2014$.

[52] R. Natalini, M. Ribot, and M. Twarogowska. A numerical comparison between degenerate parabolic and quasilinear hyperbolic models of cell movements under chemotaxis. Journal of Scientific Computing, 63(3):654-677, 2015.

[53] S. Noelle, Y. Xing, and C.-W. Shu. High-order well-balanced finite volume WENO schemes for shallow water equation with moving water. J. Comput. Phys., 226(1):29-58, 2007.

[54] E. F. Toro. Riemann solvers and numerical methods for fluid dynamics. Springer-Verlag, Berlin, third edition, 2009. A practical introduction.

[55] E.F. Toro, M. Spruce, and W. Speares. Restoration of the contact surface in the HLL-Riemann solver. Shock waves, 4(1):25-34, 1994.

[56] M. Twarogowska. Numerical approximation and analysis of mathematical models arising in cells movement. $\mathrm{PhD}$ thesis, Università degli studi de l'Aquila, 2012.

[57] Y. Xing. Exactly well-balanced discontinuous Galerkin methods for the shallow water equations with moving water equilibrium. J. Comput. Phys., 257(part A):536-553, 2014. 